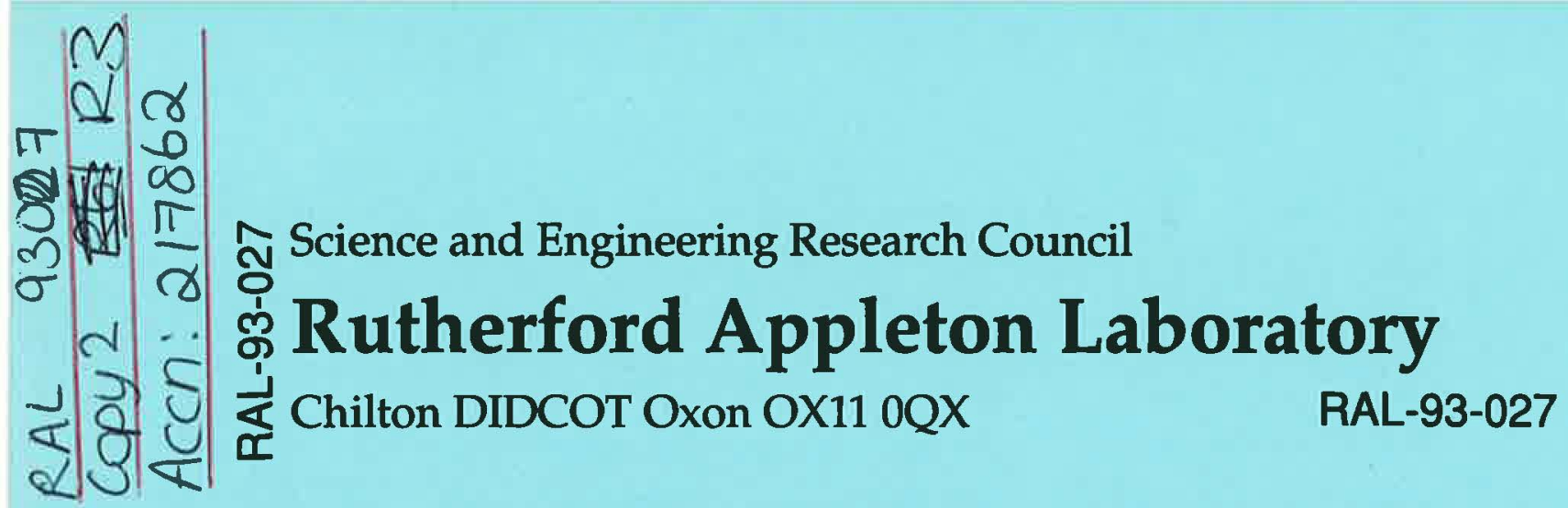

\title{
Present Status of Parton Distributions
}

A D Martin W J Stirling and R G Roberts

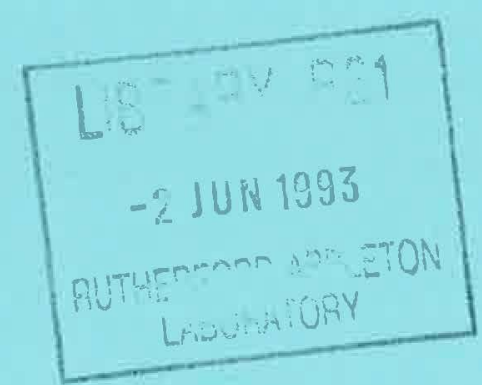

April 1993 
Science and Engineering Research Council "The Science and Engineering Research Council does not accept any responsibility for loss or damage arising from the use of information contained in any of its reports or in any communication about its tests or investigations" 


\title{
Present Status of Parton Distributions ${ }^{1}$
}

\author{
A.D. Martin and W.J. Stirling \\ Department of Physics, University of Durham \\ Durham DH1 3LE, England \\ and \\ R.G. Roberts \\ Rutherford Appleton Laboratory, \\ Chilton, Didcot OX11 OQX, England
}

\begin{abstract}
We review the present knowledge of the partonic structure of the proton. We compare and contrast the parton distributions obtained recently by the MRS and CTEQ collaborations in global analyses of deep-inelastic and related data, including the new NMC and CCFR structure function measurements. We discuss the information that is at present available on the flavour asymmetry of the sea quark distributions of the proton and, in particular, describe how well experiments can discriminate between $\bar{u}, \bar{d}$ and $\bar{s}$. We comment on extrapolations into the small $x$ region that is accessible at HERA.
\end{abstract}

\footnotetext{
${ }^{1}$ Based on an invited introductory talk given by A.D. Martin at the Workshop on 'HERA - the new frontier for QCD', Durham, March 22-26, 1993; to appear in the Proceedings of the Workshop, J. Phys. G
} 


\section{Introduction}

The distributions of the quarks (and gluons) within the proton are determined by values of the structure functions $F_{i}\left(x, Q^{2}\right)$ measured in muon (or electron)-nucleon (or nucleus) and neutrino-nucleus deep inelastic scattering experiments. For example, the relation between the observable structure function $F_{2}^{\mu p}$ and the parton distributions $f_{i}$ is of the form

$$
F_{2}^{\mu p}\left(x, Q^{2}\right)=\sum_{q} e_{q}^{2} x f_{q}\left(x, Q^{2}\right)+\alpha_{s}\left(Q^{2}\right) \sum_{i=q, g} x \int_{x}^{1} \frac{d z}{z} c_{i}(z) f_{i}\left(\frac{x}{z}, Q^{2}\right)
$$

at next-to-leading order (NLO) in $\alpha_{s}$, where the coefficients $c_{i}(z)$ are known, but scheme dependent, functions. As usual $x$ and $Q^{2}$ are the independent invariant variables that can be constructed from the 4-momenta of the proton, $p$, and the probe, $q$; that is $Q^{2} \equiv-q^{2}$ and $x \equiv-q^{2} / p \cdot q$, see Fig. 1 . Fig. 1 displays possible partonic structure of the proton as seen by the probe, $q$. Recall that it can be readily shown that $x$ is the fraction of the proton's (light-cone) momentum that is carried by the struck quark. It is useful to remember that the (incoherent) sum of the possible chains connecting the "valence" and "struck" quarks of Fig. 1 gives rise to a power series in $\alpha_{s}\left(Q^{2}\right) \log Q^{2}$ and it is this resummation which leads to the introduction of the $Q^{2}$ dependence of the $f_{i}\left(x, Q^{2}\right)$ which is governed by the Altarelli-Parisi equations. There remain non-logarithmic $O\left(\alpha_{s}\right)$ terms, as shown in eq. (1).

It is relevant to ask why we should continue to devote so much effort to the study of the distributions of partons in the proton. There are two main reasons - one experimental and one theoretical. First, a detailed knowledge of parton distributions is an essential ingredient in all "hard" interactions involving protons, and so they are needed to estimate the production rates of the various "hard" processes that may occur at current and forthcoming colliders. However the parton structure is interesting in its own right. In particular, novel perturbative QCD effects are expected to become apparent at small $x$ (i.e. $x \lesssim 10^{-3}$ ). The reason is that at small $x$ the sum over gluon chains (like the one in Fig. 1) leads to a power series in $\alpha_{s} \log (1 / x)$, which on resummation, via the Lipatov (or BFKL) equation [1], suggests that the gluon behaves as

$$
x g \sim x^{-\lambda}
$$

as $x \rightarrow 0$, with $\lambda$ predicted, typically, to be about 0.5 . Such an increase in $x g\left(x, Q^{2}\right)$, as $x$ decreases, cannot go on indefinitely. If the density of gluons becomes too large they can no longer be treated as free partons, and the effects of recombination or shadowing must be included. These small $x$ effects are addressed in subsequent introductory talks [2] and are one of the major topics of the Workshop [3]. 


\section{Survey of parton determinations}

There is a long history of determining parton distributions from deep-inelastic data. Table 1 lists just the next-to-leading order analyses, together with the data fitted. The most significant recent (pre-HERA) experimental developments are the new NMC measurements [4] of $F_{2}^{\mu p}$ and $F_{2}^{\mu n}$ (or rather $F_{2}^{\mu D}$ ) and the CCFR measurements [5] of $F_{2}^{\nu N}$ and $x F_{3}^{\nu N}$. They have had a profound impact on our knowledge of parton distributions, especially for $x \lesssim 0.1$. Only the MRS('92) and CTEQ('93) analyses (that is the three below the line in Table 1) were able to incorporate these new data, and so should be able to provide a more reliable basis for extrapolation to smaller $x$. Figure 2 serves to highlight the problems associated with the extrapolation. It shows (dashed curves) an earlier KMRS global fit [7] which included the BCDMS data, together with the new MRS('92) fit to both BCDMS and the new NMC data sets. We see that extrapolations of the old solutions considerably undershoot the new data at small $x$. The new quark distributions are therefore much larger at small $x$. On the other hand for $x \gtrsim 0.1$ there is no change; we see both the "old" and "new" $\mu p$ data and the partons are all in excellent agreement with each other.

As is apparent from Table 1 , the parton distributions, $f_{i}$, are determined from global fits to a wide range of deep inelastic and related data. The basic procedure is to parametrize the $f_{i}$ at a sufficiently large $Q_{0}^{2}$, say 4 or $5 \mathrm{GeV}^{2}$, so that $f_{i}\left(x, Q^{2}\right)$ can be calculated at higher $Q^{2}$ from perturbative QCD via the NLO Altarelli-Parisi equations. The deep-inelastic muon and neutrino data pin down the valence and sea quark distributions, but hardly constrain the gluon distribution, which only enters directly at NLO; essentially the only constraint is the momentum sum rule which shows that the gluon carries just less than $50 \%$ of the proton's momentum at $Q_{0}^{2}$. On the other hand the gluon enters at leading order in prompt photon production. Indeed for "large" $p_{T}$ photons produced by $p p \rightarrow \gamma X$, the dominant QCD subprocess is $g q \rightarrow \gamma q$, in contrast to $p \bar{p} \rightarrow \gamma X$ where the annihilation process $q \bar{q} \rightarrow \gamma g$ is much more important. The relevant data are from the WA70 collaboration [8] which determine the gluon in the region $x \sim 0.35$. Combined with the momentum sum rule constraint, this gives an input gluon behaviour $\propto(1-x)^{5.3}$ at large $x$. Data for the Drell-Yan $p N \rightarrow \mu^{+} \mu^{-} X$ process, which is mediated at LO by $q_{v a l} \bar{q}_{\text {sea }} \rightarrow \gamma^{*}$, constrain the $(1-x)^{\eta_{*}}$ behaviour of the sea quark distributions. Finally data on $W$ and $Z$ production at $\bar{p} p$ colliders impose tight constraints on the $u$ and $d$ distributions, particularly when the accurate NMC measurements of $F_{2}^{\mu n} / F_{2}^{\mu p}$ have to be fitted simultaneously. 


\begin{tabular}{|c|c|c|c|c|c|}
\hline & $\mu$-DIS & $\nu$-DIS & Prompt $\gamma$ & D-Yan & $W, Z$ \\
\hline MRS '88 & $\mathrm{EMC}+.$. & CDHSW & $\operatorname{AFS}(+J / \psi)$ & - & - \\
\hline DFLM ' 88 & $(\mathrm{EMC}+.)$. & CHARM..+ & - & $\mathrm{E} 288+.$. & - \\
\hline ABFOW ' 89 & BCDMS & - & WA70 & - & - \\
\hline HMRS '90 & $\begin{array}{l}\text { EMC } \\
\text { BCDMS } \\
\operatorname{NMC}(n / p)\end{array}$ & CDHSW & WA70 & E605 & - \\
\hline MT '90 & $\begin{array}{l}\text { EMC } \\
\text { BCDMS }\end{array}$ & CDHSW & - & $\begin{array}{l}\text { E288 } \\
\text { E605 }\end{array}$ & - \\
\hline $\begin{array}{l}\text { KMRS '90 } \\
\left.\text { (sets } B_{0}, B_{-}\right)\end{array}$ & $\begin{array}{l}\operatorname{BCDMS} \\
\operatorname{NMC}(n / p)\end{array}$ & CDHSW & WA70 & E605 & - \\
\hline $\begin{array}{l}\text { MRS (Apr '92) } \\
\left.\text { (sets } D_{0}, D_{-}\right)\end{array}$ & $\begin{array}{l}\text { BCDMS } \\
\operatorname{NMC}(p, n)^{\dagger}\end{array}$ & $\begin{array}{l}\text { CDHSW } \\
\text { CCFR }^{\dagger}\end{array}$ & WA70 & E605 & $\begin{array}{l}\text { (UA2, } \\
\text { CDF }\end{array}$ \\
\hline $\begin{array}{l}\text { MRS (Nov '92) } \\
\text { (sets } D_{0}^{\prime}, D_{-}^{\prime} \text { ) }\end{array}$ & $\begin{array}{l}\text { BCDMS } \\
\operatorname{NMC}(p, n)\end{array}$ & CCFR & WA70 & E605 & $\begin{array}{l}\text { (UA2, } \\
\text { CDF) }\end{array}$ \\
\hline CTEQ ('93) & $\begin{array}{l}\text { BCDMS } \\
\operatorname{NMC}(p, n)\end{array}$ & CCFR & $\begin{array}{l}\text { WA70 } \\
\text { E706,UA6 }\end{array}$ & E605 & \\
\hline
\end{tabular}

Table 1: NLO determinations of parton distributions $[6,7,9,10,11]$ together with the data used in the various analyses. Data marked ${ }^{\dagger}$ were used in preliminary form. 


\section{The MRS('92) analysis}

The MRS analysis [10] shows that the above wide range of data are well described by remarkably simple parametrizations of parton distributions at $Q_{0}^{2}=4 \mathrm{GeV}^{2}$; in total only about 15 parameters are required. The quality of the fit to the recent high precision deep-inelastic data is shown in Figs. 2-5. The only possible blemish is that the (renormalized) CCFR $F_{2}^{\nu N}$ data are undershot at the smallest values of $x$, that is for $x \lesssim 0.1$ (see Fig. 4). In order to provide an indication of the possible range of extrapolations to smaller values of $x$, two sets of partons are presented which describe the data equally well - one set omitting and one set including the Lipatov behaviour, (2), of the gluon and sea quark distributions at small $x$. Namely

$$
\begin{array}{ll}
x g\left(x, Q_{0}^{2}\right) \sim \text { constant } & \text { for the } D_{0}^{\prime} \text { set }, \\
x g\left(x, Q_{0}^{2}\right) \sim x^{-\frac{1}{2}} & \text { for the } D_{-}^{\prime} \text { set },
\end{array}
$$

as $x \rightarrow 0$; and similarly for $x \bar{q}\left(x, Q_{0}^{2}\right)$ since the sea is driven at small $x$ by $g \rightarrow q \bar{q}$.

Motivated by the NMC results for the Gottfried sum rule (see (11) - (13) below), we $[9,10]$ allow flavour asymmetry of the non-strange sea. Indeed from the lack of Regge $f_{2}-a_{2}$ exchange degeneracy we expect

$$
\bar{d}-\bar{u}=A x^{\beta}(1-x)^{\eta_{s}},
$$

where the behaviour at small $x$ is governed by the intercept of the meson Regge trajectory, so that $\beta \simeq-\alpha_{R}(0) \simeq-0.5$. We parametrize $\bar{d}-\bar{u}$ in this way, and for the strange sea distribution we use

$$
s=\frac{1}{4}(\bar{u}+\bar{d})
$$

at $Q_{0}^{2}$, where the (factor of 2) suppression is motivated by the CCFR results [14] for $\nu N \rightarrow \mu^{+} \mu^{-} X$.

\section{Comparison of MRS('92) and CTEQ('93) anal- yses and the strange sea}

An independent parton distribution analysis, based on essentially the same data sets, has recently been completed by the CTEQ collaboration [11]. An important difference is that they use more complicated parametric forms (employing a total of more than 30 parameters); in particular they allow the sea quark distributions $\bar{u}, \bar{d}$ and $s$ to be freely and independently parametrized. The MRS and CTEQ distributions are compared at $Q^{2}=20 \mathrm{GeV}^{2}$ in Fig. 6, where the more complicated behaviour of the CTEQ sea is apparent. Leaving aside the differences at small $x$ for the moment, it is 
informative to first compare the partons in the region of precise data. Fig. 7 shows the result of overlaying the two sets at $x=0.15$. The differences are larger than anticipated in view of the accuracy of the data.

The origin of the differences can be explained by expressing (to leading order) the observed muon and neutrino deep-inelastic structure functions in terms of the parton densities

$$
\begin{aligned}
F_{2}^{\mu p}-F_{2}^{\mu n} & =\frac{1}{3} x(u+\bar{u}-d-\bar{d}) \\
\frac{1}{2}\left(F_{2}^{\mu p}+F_{2}^{\mu n}\right) & =\frac{5}{18} x\left(u+\bar{u}+d+\bar{d}+\frac{4}{5} s\right) \\
F_{2}^{\nu N}=F_{2}^{\bar{\nu} N} & =x(u+\bar{u}+d+\bar{d}+2 s) \\
\frac{1}{2} x\left(F_{3}^{\nu N}+F_{3}^{\bar{\nu} N}\right) & =x(u-\bar{u}+d-\bar{d})
\end{aligned}
$$

where $N$ is an isoscalar nuclear target, and where we have included the $s$ quark, but neglected the (small) $c$ quark contribution. The four observables do not determine the five distributions $u, d, \bar{u}, \bar{d}$ and $s$, but only four combinations, which we can take to be $u+\bar{u}, d+\bar{d}, \bar{u}+\bar{d}$ and $s$. Inspection of Fig. 7 shows these combinations agree much better between the two analyses, as indeed they should. Although the above LO discussion is very simplistic the message is clear. Deep-inelastic data at a given $x$ and $Q^{2}$ do not determine $\bar{u}\left(x, Q^{2}\right)$ and $\bar{d}\left(x, Q^{2}\right)$ separately, but only the sum.

The differences between the MRS [10] and CTEQ [11] partons originate primarily from the different treatment of the sea quark distributions. As mentioned above, MRS use an economical parametrization based on eqs. (4) and (5). In contrast, in the CTEQ analysis the $\bar{u}, \bar{d}$ and $s$ distributions are freely and independently parametrized. As a consequence, their $s$ distribution becomes larger than both $\bar{u}$ and $\bar{d}$ for $x \lesssim 0.1$ in order to better describe the CCFR $F_{2}^{\nu N}$ data at small $x$ (see eq. (8) and recall from Fig. 4 that MRS undershoot these data). Indeed to demonstrate the need for a large strange sea CTEQ consider the structure function difference

$$
x s(x) \simeq \frac{5}{6} F_{2}^{\nu N}(x)-3 F_{2}^{\mu D}(x),
$$

which follows from (7) and (8). In Fig. 8 we show our determination of this structure function difference which includes (i) the 0.95 renormalization of the $F_{2}^{\nu N}$ data found in the MRS global analysis [10], (ii) the heavy target correction and uncertainty and (iii) deuteron screening corrections. If this was the only information about $s(x)$ we would conclude that the MRS input assumption, (5), of a factor of 2 suppression of the strange sea was not valid. However there is an independent and more direct measure of the strange sea, namely the process $\nu N \rightarrow \mu^{-} \mu^{+} X$ for which the dominant QCD subprocess is $\nu s \rightarrow \mu^{-}\left(c \rightarrow \mu^{+}\right)$. Indeed we have mentioned that the original CCFR dimuon data were the basis of (5). There are more recent CCFR dimuon data [15] from which they determine the values of $x s(x)$ shown in Fig. 9, which suggest that the strange sea is more than a factor of 2 suppressed. Actually they find the 
extra suppression of $s(x)$ arises from a large value of the non-strange sea which they extract from their single muon structure function measurements [5] at small $x$ the very data that MRS undershoot and that CTEQ claim requires a considerably enhanced $s(x)$. In fact the quantity $\eta \equiv 2 S /(U+D)$ (where $U \equiv \int_{0}^{1} x u(x) d x$ etc.), which is more reliably measured in dimuon experiments, is found by CCFR [15] to be $\eta=0.064_{-0.007}^{+0.008} \pm 0.002$ at $Q^{2}=22 \mathrm{GeV}^{2}$ (a value essentially unchanged from their earlier analysis). This agrees well with the $\operatorname{MRS}\left(\mathrm{D}_{0}^{\prime}\right)$ prediction of $\eta=0.066$ [10].

The conflict of the two different experimental determinations of $x s(x)$ is evident if we overlay Figs. 8 and 9. It appears that the conservative MRS input assumption, (5), (corresponding to the continuous curves) gives the most reasonable compromise at present. (Indeed it is interesting to note that if the CCFR measurements of $F_{2}^{\nu N}$ were reduced at small $x$, then the data points on Fig. 8 would fall, while those on Fig. 9 would rise!) On the other hand, from all points of view, the CTEQ strange sea looks too large for $x \lesssim 0.1$.

\section{$5 \quad$ The non-strange sea: probing $\bar{u} \neq \bar{d}$}

We have seen that the deep-inelastic data do not determine $\bar{u}$ and $\bar{d}$ separately. In fact, prior to 1992, analyses assumed that the non-strange sea was flavour symmetric, that is $\bar{u}=\bar{d}$. The motivation for allowing $\bar{u} \neq \bar{d}$ came from comparing the NMC measurement [16]

$$
\int_{0.004}^{0.8} \frac{d x}{x}\left(F_{2}^{\mu p}-F_{2}^{\mu n}\right)=0.227 \pm 0.007 \text { (stat.) } \pm 0.014 \text { (sys.) }
$$

at $Q^{2}=4 \mathrm{GeV}^{2}$ with the Gottfried-sum-rule expectation

$$
\begin{aligned}
I_{G S R}=\int_{0}^{1} \frac{d x}{x}\left(F_{2}^{\mu p}-F_{2}^{\mu n}\right) & =\frac{1}{3} \int_{0}^{1} \frac{d x}{x}\left(u_{V}-d_{V}\right)+\frac{2}{3} \int_{0}^{1} \frac{d x}{x}(\bar{u}-\bar{d})(12 \text { if } \bar{u}=\bar{d} \\
& =\frac{1}{3}, \quad
\end{aligned}
$$

A straightforward comparison of (11) and (13) implies $\bar{u} \neq \bar{d}$, and in refs. $[9,10]$ we parametrized $\bar{d}-\bar{u}$ according to (4) and found $\bar{d}>\bar{u}$ such that $I_{G S R}$ was in the range $0.24-0.26$. It is interesting to note that, even including the NMC data, it is still possible to maintain $\bar{u}=\bar{d}$ and to obtain an equally good global description of the data at the expense of a contrived small $x$ behaviour of the valence distributions, see set $S_{0}$ and especially the discussion in [9]. However, we concluded that it is more physical to allow $\vec{u} \neq \bar{d}$ and hence we introduced sets $\mathrm{D}_{0}$ and $\mathrm{D}_{-}$.

The structure of $\bar{d}-\bar{u}$ is not determined by available data. All we really know is that on average $\bar{d}>\bar{u}$ at small $x$. The situation is summarized in Fig. 10 which shows the ingredients of the Gottfried sum rule for various sets of partons with $\bar{u} \neq \bar{d}$. 


\begin{tabular}{|l|ccc|}
\hline set & $g$ & $\bar{u}$ & $\bar{d}$ \\
\hline MRS(D $\left.D_{0}^{\prime}\right)$ & $(0,0)$ & $(0,0)$ & $(0,0)$ \\
MRS(D'- & $(-0.5,0)$ & $(-0.5,0)$ & $(-0.5,0)$ \\
CTEQ1M & $(0.179,2.338)$ & $(-0.032,0)$ & $(-0.158,0)$ \\
CTEQ1MS & $(-0.384,0.093)$ & $(0.028,0)$ & $(-0.273,0)$ \\
\hline
\end{tabular}

Table 2: Coefficients $(a, b)$ for the analytic behaviour $x f \sim x^{a} \log (1 / x)^{b}$ as $x \rightarrow 0$ at $Q_{0}^{2}=4 \mathrm{GeV}^{2}$, for the $\operatorname{MRS}\left(\mathrm{D}_{0}^{\prime}, \mathrm{D}_{-}^{\prime}\right)$ and $\mathrm{CTEQ}(1 \mathrm{M}, 1 \mathrm{MS})$ gluon and sea quark distributions.

The central part of the figure is a visual display of the integrand of the sum rule (12). The data are from NMC [18]. The top part of the figure shows the accumulated contribution to the sum rule as we integrate down from $x=1$; thus the limiting values of the curves as $x \rightarrow 0$ give $I_{G S R}$ of (12). The central curves in the shaded bands correspond to $\operatorname{MRS}\left(\mathrm{D}_{0}^{\prime}\right)$ partons [10] with $\bar{d}-\bar{u}$ parametrized as in (4); these partons give $I_{G S R}=0.256$. To explore variations about this solution we repeated [17] the global analysis with parametrization (4) extended to

$$
\bar{d}-\bar{u}=A x^{\beta}(1-x)^{\eta_{s}}\left(1+\gamma x+\delta x^{2}\right)
$$

with $A$ chosen to maintain $I_{G S R}=0.256$. The shaded band corresponds to acceptable fits with $\gamma$ in the range $-8<\gamma<32$ and $\delta=0$. We see that $F_{2}^{\mu p}-F_{2}^{\mu n}$ is only modified for $x \lesssim 0.05$; indeed deep inelastic data impose only a weak constraint on $\bar{d}-\bar{u}$ at larger values of $x$.

How can we determine $\bar{d}-\bar{u}$ for $x \gtrsim 0.05$ ? One suggestion [19] is to compare Drell-Yan production of $p p$ and $p n$ origin. A hint of what may be possible is shown in Fig. 11, which compares Drell-Yan data [20] obtained from a neutron rich (tungsten) target with those from isoscalar targets. In addition to partons with $-8<\gamma<32$ and $\delta=0$ (shaded band), the dashed curves in Figs. 10 and 11 give the results of a global fit with $\gamma=-60$ and $\delta=300$ chosen simply to reproduce the qualitative trend of the Drell-Yan data of Fig. 11. This fit has a different $\bar{d}-\bar{u}$ shape and, in fact, the quality of the overall fit to the deep-inelastic data diminishes.

For completeness we also show the CTEQ1M prediction (dot-dashed curves) on Figs. 10 and 11. The dramatically different behaviour is a consequence of freely parametrized $\bar{u}$ and $\bar{d}$ distributions [11]; in particular the results for the Gottfried sum rule reflect the $x \rightarrow 0$ behaviour $x(\bar{d}-\bar{u}) \sim x^{-0.16}$ (see Table 2) in contrast to imposing the Regge expectation $x(\bar{d}-\bar{u}) \sim x^{0.5}$ of (4).

Fig. 12 shows the predictions for the asymmetry between Drell-Yan production 
on proton and neutron targets

$$
A_{D Y}=\frac{\sigma_{p p}-\sigma_{p n}}{\sigma_{p p}+\sigma_{p n}}
$$

as a function of $\sqrt{\tau}$, at $y=0$. Because $u>d$ in the proton, the asymmetry is positive for sets with $\bar{d}-\bar{u}$ zero or small at large $x$. A measurement [21] of the asymmetry to an accuracy of a few per cent will provide a powerful discriminator of the possible behaviours of $\bar{d}-\bar{u}$.

\section{Extrapolations into the small $x$ HERA regime}

The precision of the available deep-inelastic and related data means that we should now have reliable sets of partons at least in the region $x \gtrsim 0.05$, provided reasonable behaviour of $\bar{d}-\bar{u}$ is assumed. Extrapolations outside the region of the data are notoriously unreliable. All we can do is to extrapolate the various sets to small $x$ and show the general trends.

Figs. 6(a) and 13(a) show the parton sets $D_{0}^{\prime}$ and $D_{-}^{\prime}$ respectively [10], where the latter set incorporate the singular Lipatov behaviour $x g, x \bar{q} \sim x^{-\frac{1}{2}}$ at small $x$, see (3). These two sets are essentially identical for $x \gtrsim 0.02$ but are dramatically different at small $x$. In particular they lead to the very different predictions for $F_{2}^{\text {ep }}$ in the HERA regime, which are shown by the continuous curves in Fig. 14. On the other hand the 'singular' CTEQ set, CTEQIMS of Fig. 13(b), turns out to be somewhat similar to the non-singular CTEQ1M solution shown in Fig. 3(b), and hence both give similar predictions for $F_{2}^{e p}$, see Fig. 14. These behaviours at small $x$ can be anticipated from the parameter values listed in Table 2; evidently for the CTEQ1MS set the singular behaviour is not imposed on the antiquark distributions, which are directly probed in $F_{2}^{e p}$.

We show in Fig. 13(c) the partons found in the "valence" model of Glück, Reya and Vogt (GRV) [12], and in Fig. 14 the corresponding prediction for $F_{2}^{\text {ep }}$. In this model, "valence" distributions at very low $Q\left(Q_{0}^{2}=0.3 \mathrm{GeV}^{2}\right)$ are evolved and fitted to MRS valence quark distributions at a higher $Q$. The growth seen for GRV at small $x$ is thus a result of Altarelli-Parisi $Q^{2}$ evolution. By coincidence, it is not unlike that seen for $D_{-}^{\prime}$, at least in the HERA regime. It is probably fair to say that much of the attractiveness of the original GRV model was lost when, besides the valence quarks (which could be regarded as of constituent origin), it was found necessary to also input valence gluon and sea quark contributions in order to respectively account for the WA70 prompt photon data [8] and to give the observed $x$ shape of the $F_{2}^{\mu p}$ data. Nevertheless, unlike $D_{-}^{\prime}$, GRV is a parameter-free prediction at small $x$.

We should add to the extrapolations shown in Fig. 14 a QCD calculation of $F_{2}^{e p}$ at small $x$ based on the "Lipatov" summation [23], see Kwiecinski [2]. One feature of this 
calculation is that the shadowing effects on $F_{2}^{e p}$ are found to be smaller than previous estimates $[7,9]$. The reason is that the calculation explicitly includes the $g \rightarrow q \bar{q}$ convolution and that the shadowing of $\bar{q}$ (and hence of $F_{2}^{e p}$ ) samples the shadowing of $g$ at a larger $x$. The results are shown in Fig. 15, together with the preliminary H1 measurements of $F_{2}^{\text {ep }}$ which were revealed in a subsequent introductory talk by De Roeck [22]. The implications of these exciting data from $\mathrm{H} 1$ became a major topic of study at the Workshop, see the summary talks [24, 3]. The preliminary verdict of Figs. 14 and 15 is that the data favour the parton sets $\mathrm{D}_{-}^{\prime}$ and GRV.

The measurements of $F_{2}^{e p}$ at HERA probe primarily the sea quark distribution at small $x$. Methods for directly extracting the gluon distribution from HERA measurements of $F_{L}$ [25] and $d F_{2} / d \log Q^{2}[26]$ have been suggested. These are based on the observation that in the leading-order expressions

$$
\begin{aligned}
F_{L}\left(x, Q^{2}\right)= & \frac{\alpha_{s}\left(Q^{2}\right)}{\pi}\left\{\frac{4}{3} \int_{x}^{1} \frac{d y}{y}\left(\frac{x}{y}\right)^{2} F_{2}\left(y, Q^{2}\right)\right. \\
& \left.+2 \sum_{q} e_{q}^{2} \int_{x}^{1} \frac{d y}{y}\left(\frac{x}{y}\right)^{2}\left(1-\frac{x}{y}\right) y g\left(y, Q^{2}\right)\right\} \\
\frac{d F_{2}\left(x, Q^{2}\right)}{d \log Q^{2}}= & \frac{\alpha_{s}\left(Q^{2}\right)}{2 \pi}\left\{\int_{x}^{1} \frac{d y}{y}\left(\frac{x}{y}\right) P_{q q}\left(\frac{x}{y}\right) F_{2}\left(y, Q^{2}\right)\right. \\
& \left.+2 \sum_{q} e_{q}^{2} \int_{x}^{1} \frac{d y}{y}\left(\frac{x}{y}\right) P_{q g}\left(\frac{x}{y}\right) y g\left(y, Q^{2}\right)\right\}
\end{aligned}
$$

it is the gluon term on the right-hand-side that dominates at small $x$ in each case. The clear correlations shown in Fig. 16 lend support to the case for making precise measurements of these two quantities, leading to qualitative estimates of the underlying gluon behaviour. At such small $x$ values, however, NLO corrections can disturb the simple formulae used to extract $x g(x)$, which are based only on $O\left(\alpha_{s}\right)$ expressions. Thus in computing the $F_{L}$ predictions shown in Fig. 16 we use the $O\left(\alpha_{s}^{2}\right)$ expressions of ref. [27], where the NLO corrections can be significant for $x \lesssim 10^{-3}$. In addition, the $O\left(\alpha_{s}\right)$ contributions to the splitting functions $P_{q g}(z)$ and $P_{q q}(z)$ involve contributions proportional to $z^{-1}$, which become increasingly important in $d F_{2} / d \log Q^{2}$ at low $x$.

It is interesting to compare the above methods for measuring the small $x$ gluon at HERA with the more direct methods available at hadron colliders. In principle, the small $x$ gluon is probed in prompt photon, large $p_{T}$ jet and $b \vec{b}$ production in high energy $p \bar{p}$ collisions, through such subprocesses as $g q \rightarrow \gamma q, g g \rightarrow g g$ and $g g \rightarrow b \bar{b}$. However, each of these methods is problematical (for a recent review see ref. [28]). Prompt photon production in the small-to-medium $p_{T}^{\gamma}$ range is plagued by uncertainties in the choice of QCD scales, the poorly known higher-order process contributions, and the matching of the experimental and theoretical "isolation 
criteria". The relatively high $p_{T}$ thresholds required for hadronic jet cross sections and the experimental uncertainties in the jet energy measurements currently prevent the small $x$ of the gluon being explored with any precision. Finally, in $b \bar{b}$ production there are large uncertainties from the significant renormalization/factorization scheme dependence and from the experimental extrapolation of the total cross section from a relatively small number of events with leptons or $J / \psi$ 's. In spite of this, it is interesting to note that there are some indications that "conventional" gluons of the form $x g \sim A(1-x)^{n}$ or similar are unable to totally explain the yield of $b \bar{b}$ pairs, in particular the CDF data at $\sqrt{s}=1.8 \mathrm{TeV}$. Berger et al. [29] have recently investigated whether it is possible to modify the gluon to achieve better agreement. It turns out to be necessary to significantly enhance the gluon in the $x \sim 0.01$ region, at the expense of poorer fits to the lower energy fixed-target prompt photon data. A precision measurement of the gluon at HERA using the methods described above may be well be the only way to settle the issue.

\section{Acknowledgements}

We thank A. De Roeck, W. Krasny and J. Kwiecinski for valuable discussions.

\section{References}

[1] E.A. Kuraev, L.N. Lipatov and V.S. Fadin, Sov. Phys. JETP 45 (1977) 199. Ya.Ya. Balitsky and L.N. Lipatov, Sov. J. Nucl. Phys. 28 (1978) 822.

[2] J. Kwiecinski, these proceedings.

E. Levin, these proceedings.

A.H. Mueller, these proceedings.

[3] A.D. Martin, small $x$ summary talk, these proceedings.

[4] NMC: P. Amaudruz et al., Phys. Lett. 295B (1992) 159.

[5] CCFR collaboration: Phys. Rev. Lett., to be published.

[6] A.D. Martin, R.G. Roberts and W.J. Stirling, Phys. Rev. D37 (1988) 1161; see also Phys. Lett. 206B (1988) 327; Mod. Phys. Lett. A4 (1989) 1135. M. Diemoz, F. Ferroni, E. Longo and G. Martinelli, Z. Phys. C39 (1988) 21. P. Aurenche, R. Baier, M. Fontannaz, J.F. Owens and M. Werlen, Phys. Rev. D39 (1989) 3275. 
P.N. Harriman, A.D. Martin, R.G. Roberts and W.J. Stirling, Phys. Rev. D42 (1990) 798.

J.G. Morfin and W.-K. Tung, Z. Phys. C52 (1991) 13.

[7] J. Kwiecinski, A.D. Martin, R.G. Roberts and W.J. Stirling, Phys. Rev. D42 (1990) 3645.

[8] WA70 collaboration: M. Bonesini et al., Z. Phys. C38 (1988) 371.

[9] A.D. Martin, W.J. Stirling and R.G. Roberts, Phys. Rev. D47 (1993) 867.

[10] A.D. Martin, W.J. Stirling and R.G. Roberts, RAL preprint-92-078 (1992), Phys. Lett. (in press).

[11] CTEQ collaboration: J. Botts, J. Morfin, J. Owens, J. Qiu, W.-K. Tung and H. Weerts, Phys. Lett. 304B (1993) 159.

[12] M. Glück, E. Reya and A. Vogt, Z. Phys. C48 (1990) 471, C53 (1992) 127; Dortmund preprint DO-TH-93-02 (1993), Phys. Lett. (in press).

[13] BCDMS collaboration: A.C. Benvenuti et al., Phys. Lett. 223B (1989) 485.

[14] CCFR collaboration: C. Foudas et al., Phys. Rev. Lett. 64 (1990) 1207.

[15] CCFR collaboration: S.A. Rabinowitz et al., Phys. Rev. Lett. 70 (1993) 134.

[16] NMC: P. Amaudruz et al., Phys. Rev. Lett. 66 (1991) 2712.

[17] A.D. Martin, R.G. Roberts and W.J. Stirling, RAL preprint RAL-93-014 (1993).

[18] NMC: E.M. Kabuß, Nucl. Phys. B (Proc. Supp.) 29A (1992) 1.

[19] S.D. Ellis and W.J. Stirling, Phys. Lett. 256B (1991) 258.

[20] E772 collaboration: P.L. McGaughey et al., Phys. Rev. Lett. 69 (1992) 1726.

[21] M.C. Abreu et al., CERN proposal SPSLC 92-15 (1992).

G.T. Garvey et al., Fermilab proposal P866 (1992).

[22] H1 collaboration: A. De Roeck, these proceedings.

[23] A.J. Askew, J. Kwiecinski, A.D. Martin and P.J. Sutton, Phys. Rev. D47 (1993) 3775 .

[24] W.J. Stirling, structure function summary talk, these proceedings.

[25] A.M. Cooper-Sarkar et al., Zeit. Phys. C39 (1988) 281. 
[26] K. Prytz, RAL preprint RAL-93-015 (1993).

[27] E.B. Zijlstra and W.L. van Neerven, Nucl. Phys. 383 (1992) 525.

[28] W.J. Stirling, Durham University preprint DTP/93/10 (1993), to appear in Proc. Conference on 'QCD - 20 Years Later', Aachen, July 1992.

[29] E.L. Berger, R. Meng and W.-K. Tung, Phys. Rev. D46 (1992) 1895.

E.L. Berger, R. Meng and J. Qiu, Argonne preprint ANL-HEP-CP-92-79 (1992). E.L. Berger and R. Meng, Argonne preprints ANL-HEP-CP-92-108 (1992), ANLHEP-PR-93-7 (1993). 


\section{Figure Captions}

1. The partonic structure of the proton as seen by a probe of 4 -momentum $q$. The struck quark carries a fraction $x$ of the proton's 4-momentum. The gluon chain may contain quark links, particularly at large $x$.

2. The BCDMS [13] and NMC [4] measurements of $F_{2}^{\mu p}$, together with the fits by KMRS [7] and MRS [10]. The NMC data were not available for KMRS. The figure is taken from ref. [10].

3. The description of the NMC data [4] for $F_{2}^{\mu n} / F_{2}^{\mu p}$ given by the $\mathrm{D}_{0}^{\prime}, \mathrm{D}_{-}^{\prime}$ and $\mathrm{S}_{0}^{\prime}$ partons [10]. The mean $Q^{2}$ of the data varies with $x$ as shown by the uppermost scale. The curves take this $Q^{2}$ dependence into account. The values of $F_{2}^{\mu n}$ have been obtained from $\mu D$ data corrected for deuteron screening effects. The figure is taken from ref. [10].

4. The continuous curves show the description of the CCFR [5] measurements of $F_{2}^{\nu N}\left(x, Q^{2}\right)$ by the $\mathrm{D}_{0}^{\prime}$ set of partons. The data are shown after correction for the heavy target effects and after the overall renormalization of 0.95 required by the global MRS fit. The statistical, systematic and heavy target correction errors have been combined in quadrature. The figure is taken from ref. [10].

5. As for Fig. 4 but for the structure function $x F_{3}^{\nu N}\left(x, Q^{2}\right)$.

6. The parton distributions of the proton at $Q^{2}=20 \mathrm{GeV}^{2}$ corresponding to (a) set $D_{0}^{\prime}$ of MRS [10] and to (b) set CTEQ1M of [11].

7. A comparison of the $\operatorname{MRS}\left(\mathrm{D}_{0}^{\prime}\right)$ and $\mathrm{CTEQ} 1 \mathrm{M}$ quark distributions (shown in Fig. 6) at $x=0.15$ and $Q^{2}=20 \mathrm{GeV}^{2}$.

8. An estimate of $x s(x)$ from structure function data $[4,5]$, together with the values found in the $\operatorname{MRS}\left(\mathrm{D}_{0}^{\prime}\right)[10]$ and CTEQ1M [11] analyses.

9. The values of $x s(x)$ determined by CCFR [15] from their dimuon deep-inelastic data, together with the values found in the $\operatorname{MRS}\left(D_{0}^{\prime}\right)$ [10] and CTEQ1M [11] analyses. The errors shown on the "data" are purely statistical.

10. The ingredients of the Gottfried sum rule for various sets of parton distributions, as described in the text. The figure is taken from ref. [17].

11. The ratio of dilepton yields per nucleon from tungsten and isoscalar targets as a function of $x$ (target). The data are from ref. [20]; the open circles at small $x$ show the ratio before correction for nuclear shadowing. The curves are the full next-to-leading order QCD predictions using the same parton sets as in Fig. 10. The figure is taken from ref. [17]. 
12. Predictions for the Drell-Yan asymmetry, (15), as a function of $\sqrt{\tau}=M / \sqrt{s}$ with $p_{\text {beam }}=450 \mathrm{GeV} / \mathrm{c}$. The curves are based on next-to-leading order calculations using the same parton distributions as in Fig. 10. The figure is taken from ref. [17].

13. The parton distributions in the proton at $Q^{2}=20 \mathrm{GeV}^{2}$ corresponding to (a) set $\operatorname{MRS}\left(\mathrm{D}_{-}^{\prime}\right)$ of [10], (b) set CTEQ1MS of [11], and (c) the "valence" model of Glück, Reya and Vogt [12]; we thank A. Vogt for the plot of GRV(1993).

14. Predictions for $F_{2}^{e p}$ at $Q^{2}=20 \mathrm{GeV}^{2}$ obtained from the parton sets $[10,11,12]$ of Figs. 6 and 13.

15. The continuous and dashed curves are the QCD predictions for $F_{2}^{e p}$ of ref. [23]. The upper and lower dashed curves correspond respectively to conventional and "hot-spot" shadowing. Also shown are the preliminary $\mathrm{H} 1$ data [22] together with the extrapolations resulting from MRS parton sets $\mathrm{D}_{0}^{\prime}$ and $\mathrm{D}_{-}^{\prime}[10]$. The data are subject to an additional overall normalization uncertainty of $\pm 12 \%$.

16. The sensitivity of two structure function methods of determining the small $x$ gluon at HERA, illustrated in terms of the gluon distributions of ref. [10]. $F_{L}$ is the longitudinal structure function for deep-inelastic $e p$ scattering. The curves are calculated using the full next-to-leading order QCD expressions. 


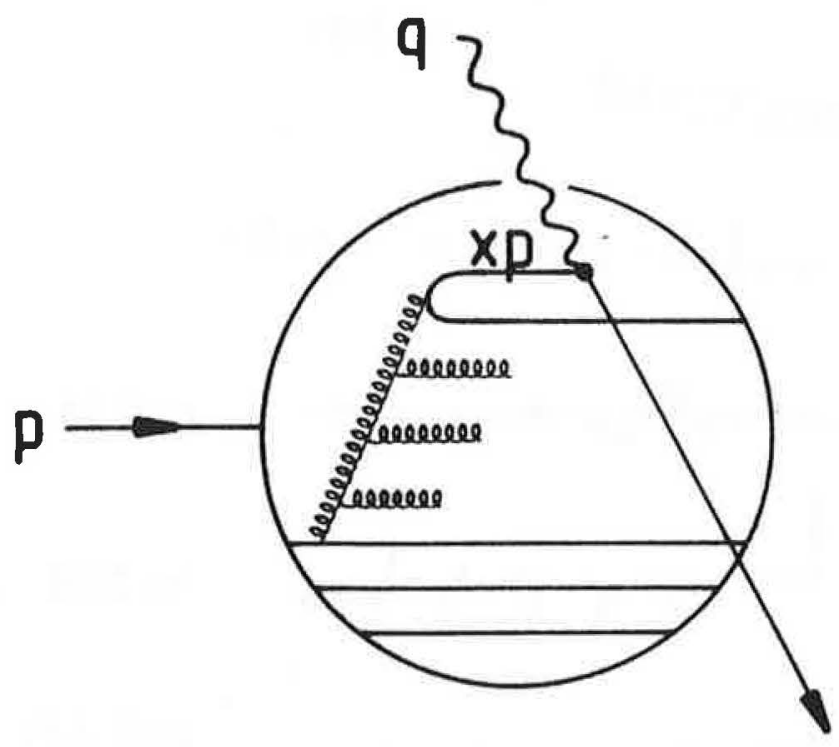

Fig. 1 

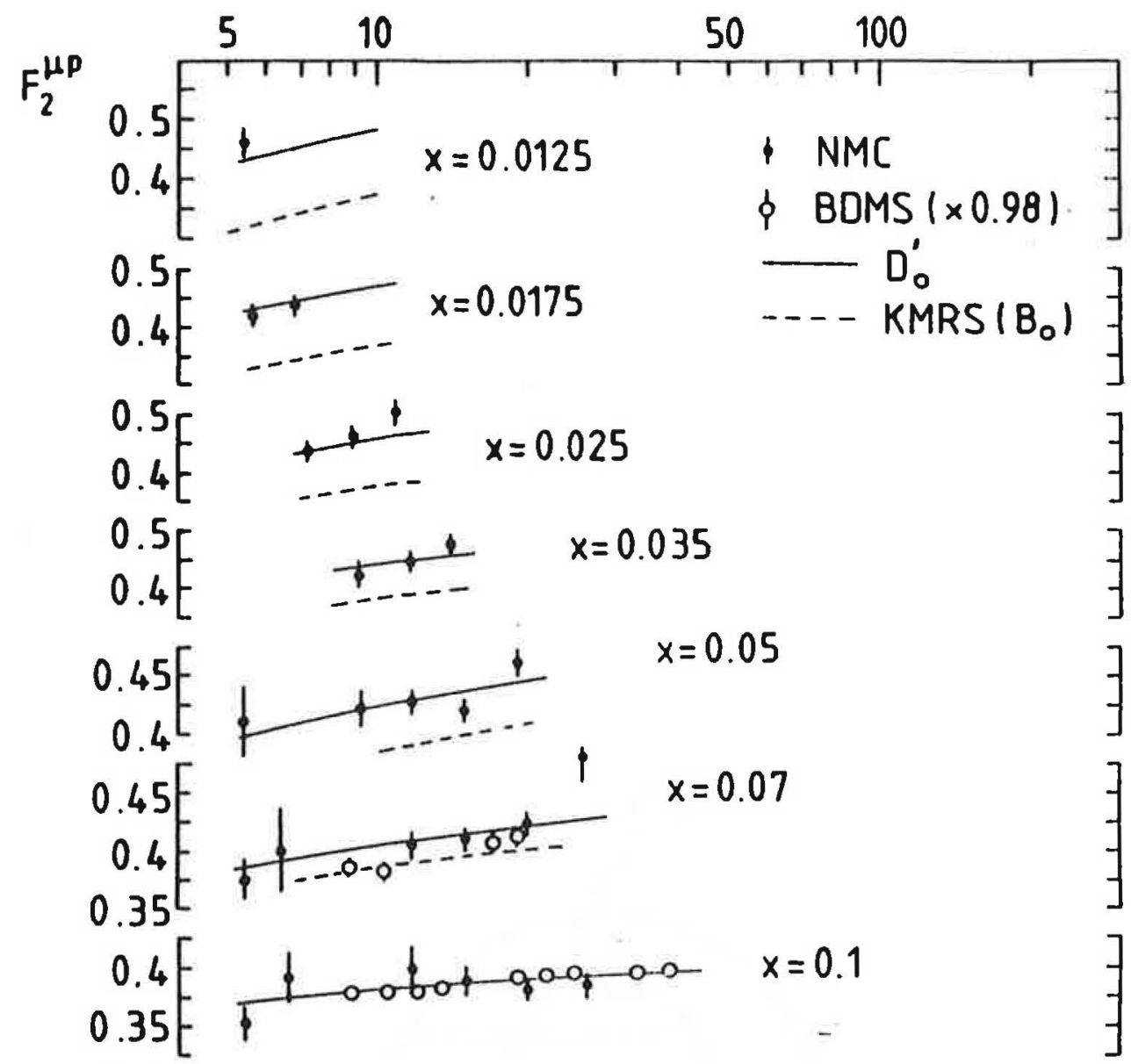

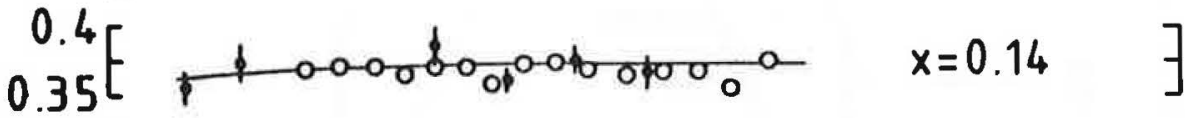

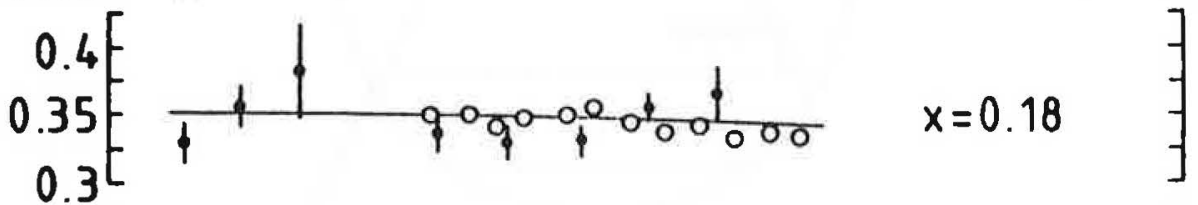

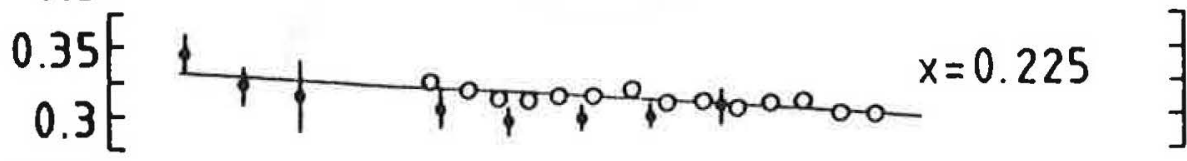
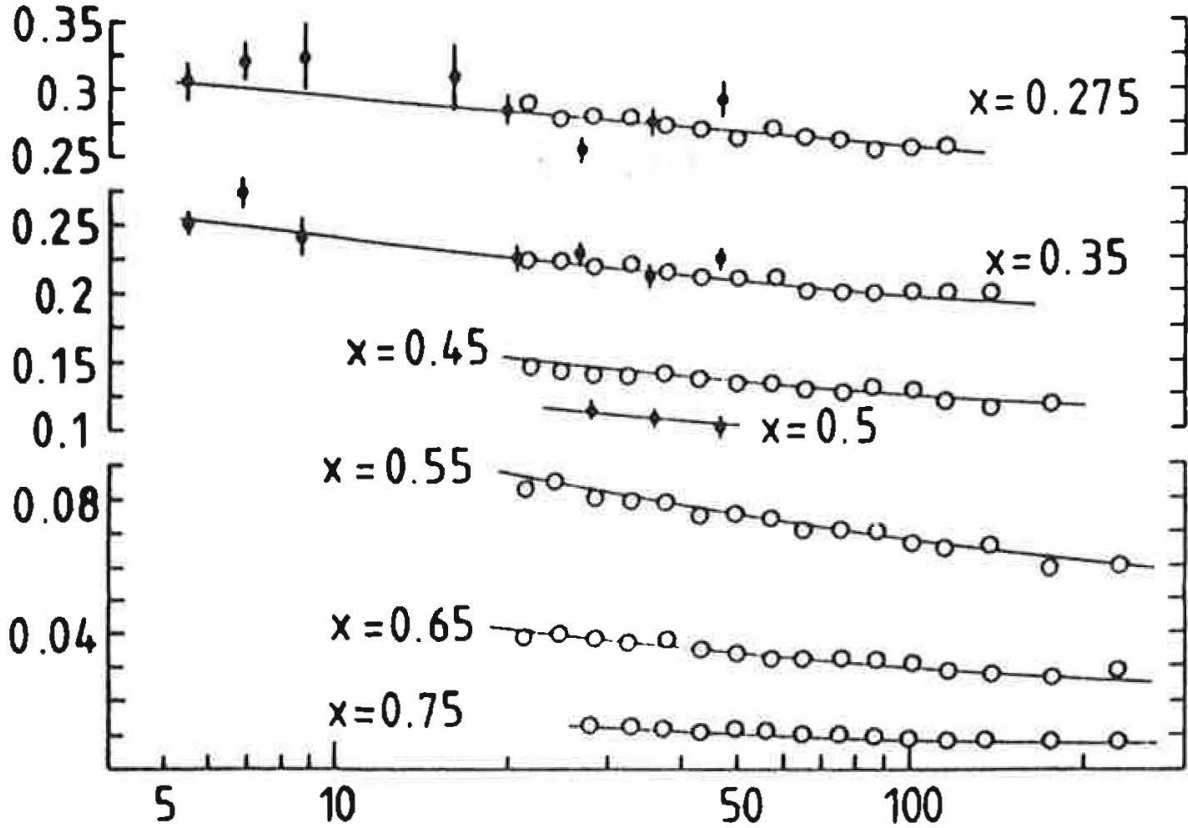

$Q^{2}\left(G e V^{2}\right) \quad$ Fig. 2 


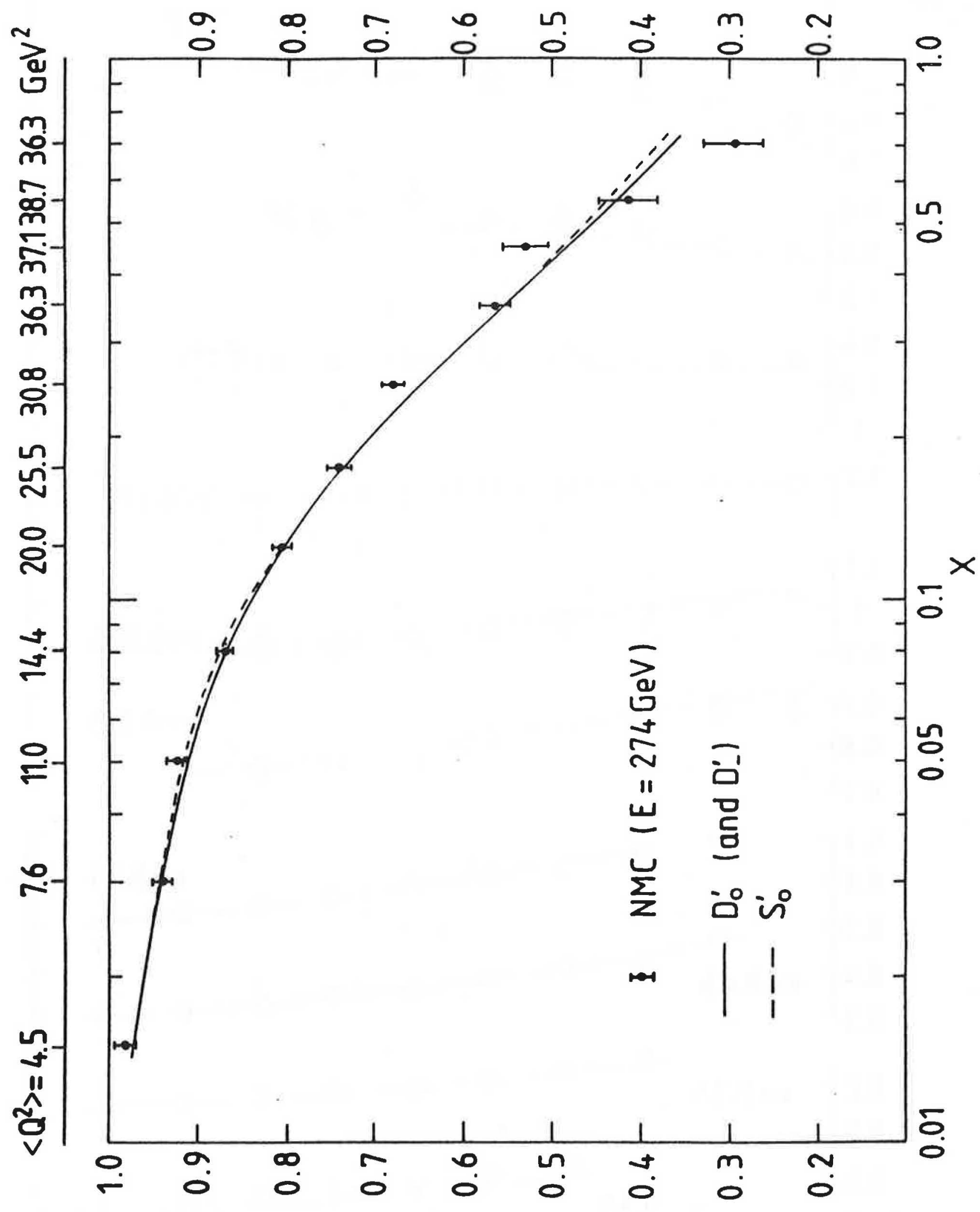

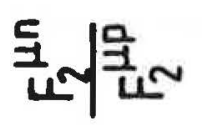

Fig. 3 


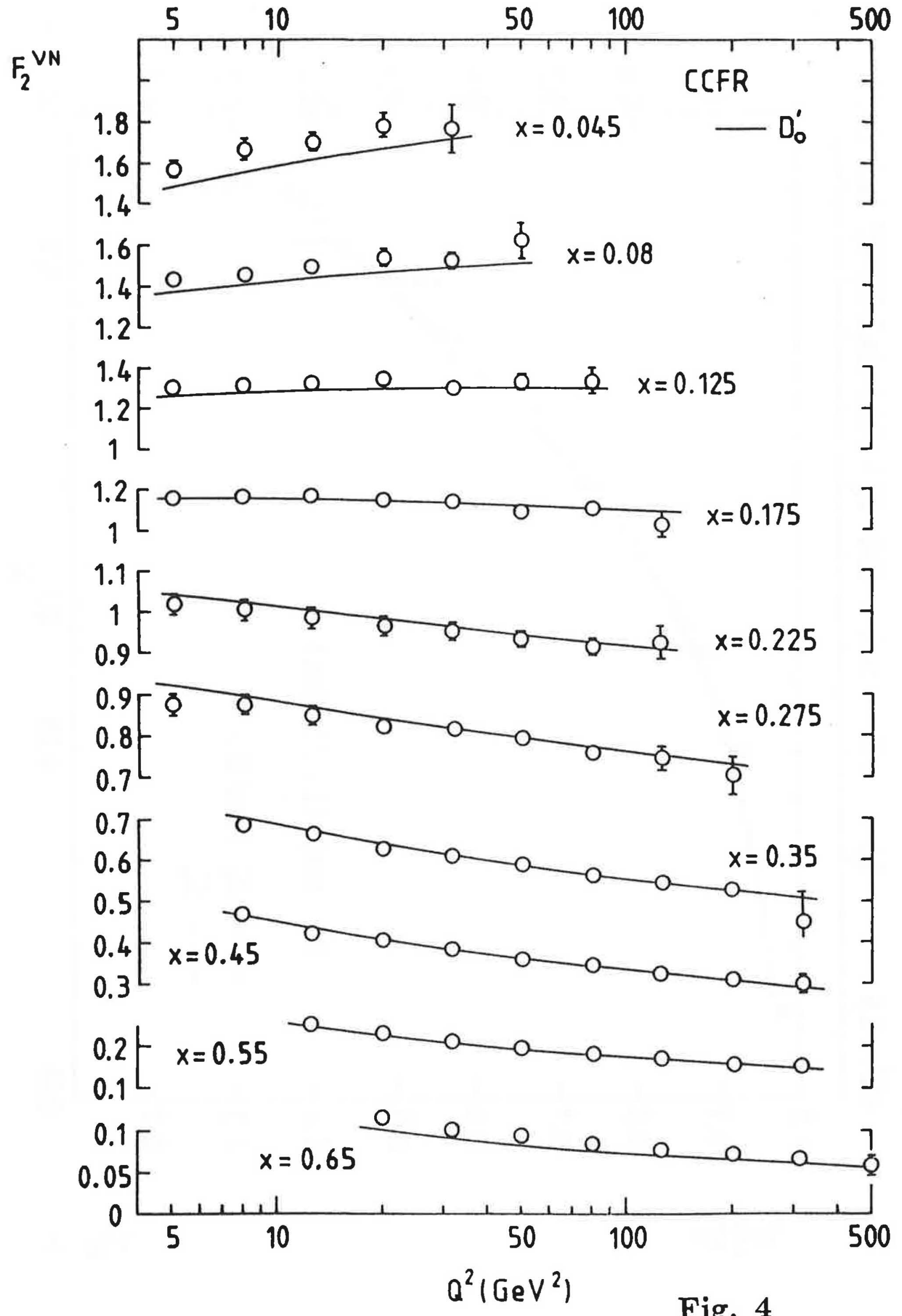

Fig. 4 
$\left\langle F_{3}^{V N}\right.$
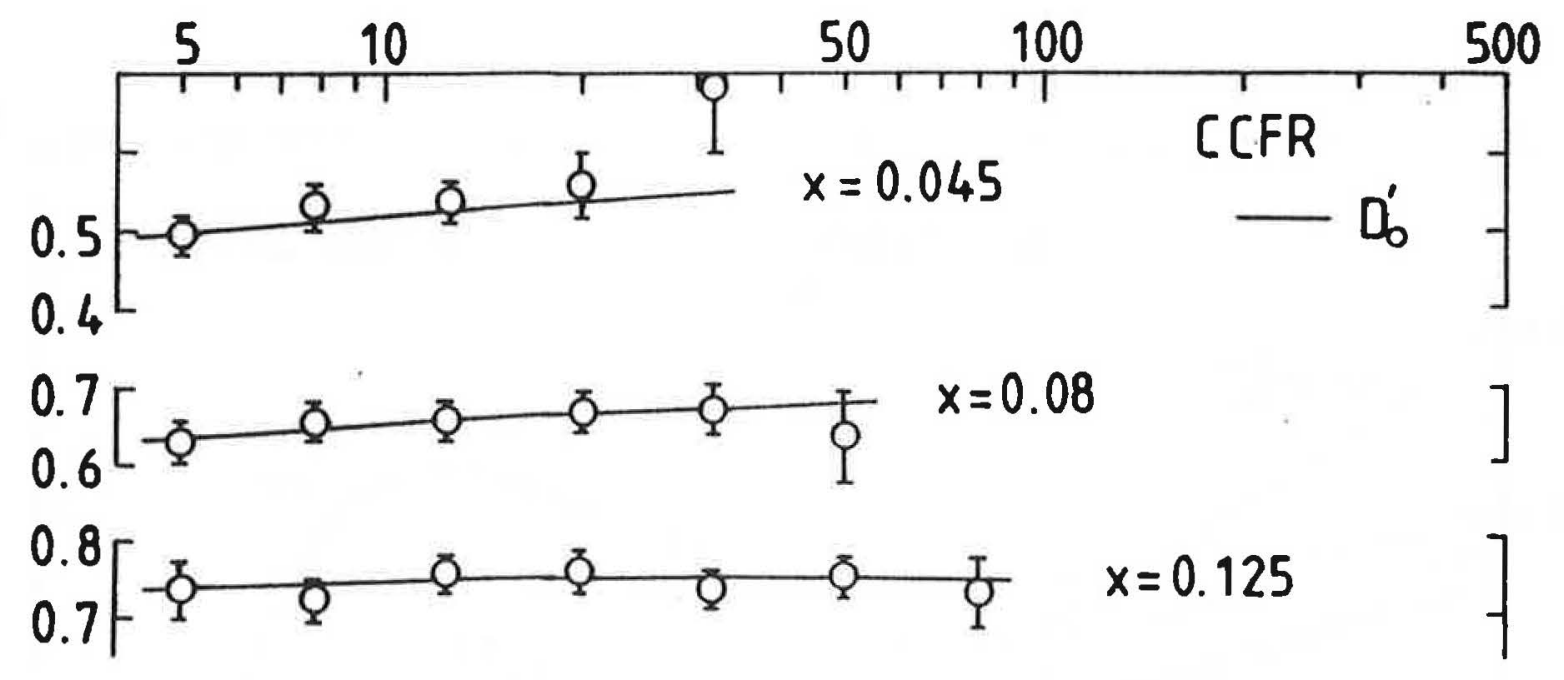

0.8
0.7
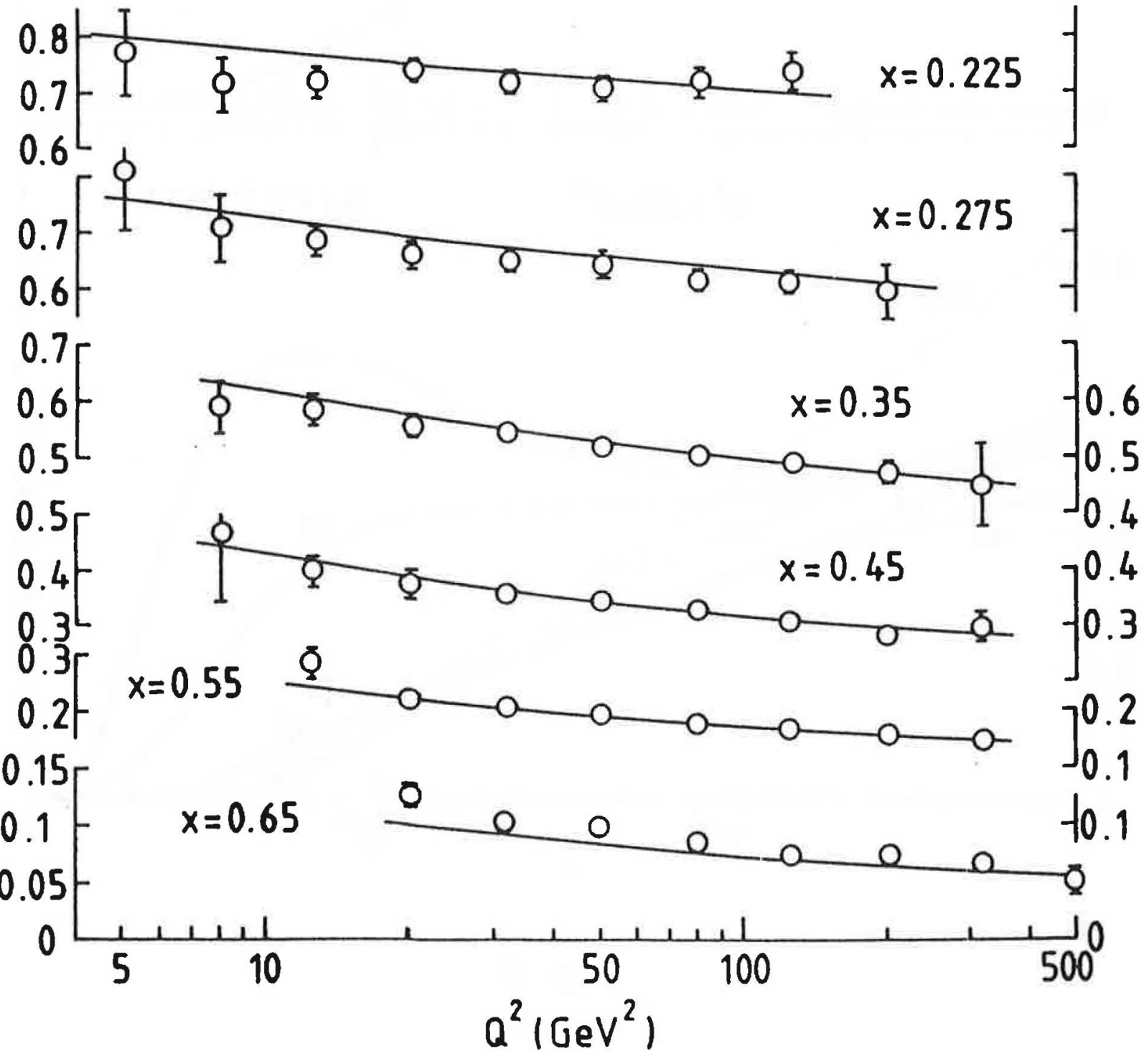

Fig. 5 

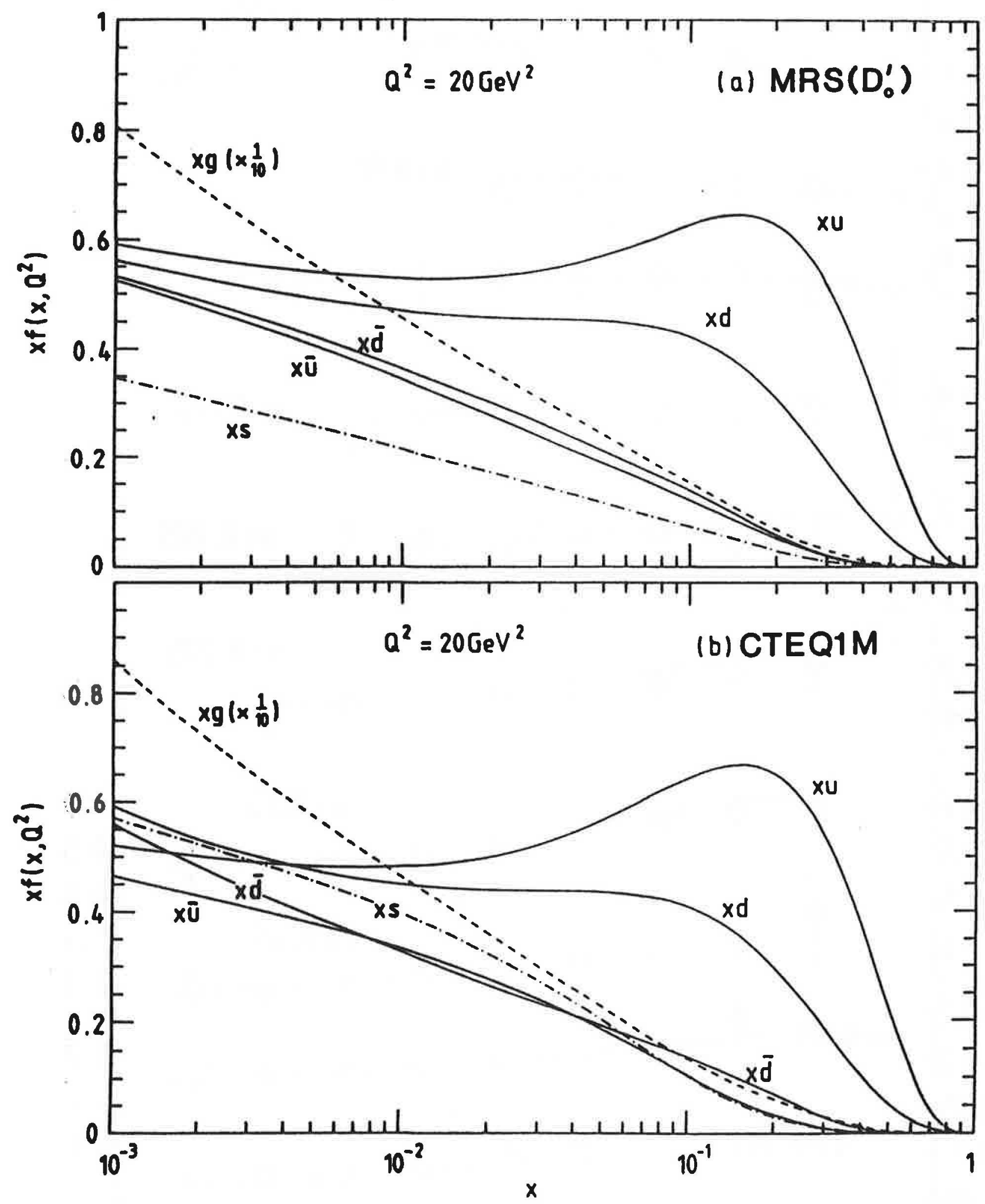

Fig. 6 


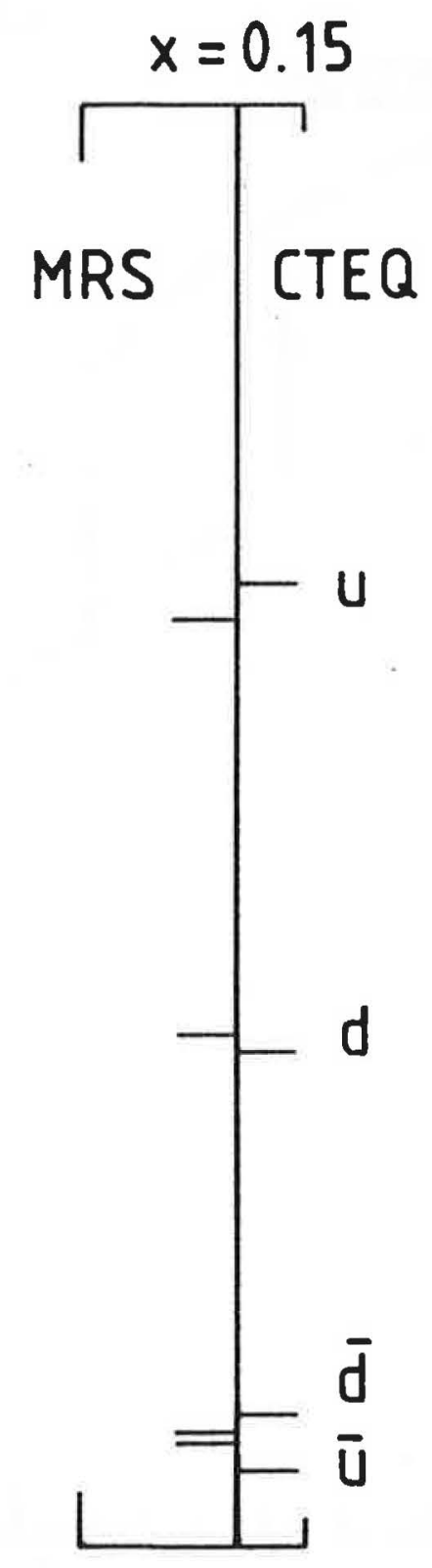

Fig. 7 


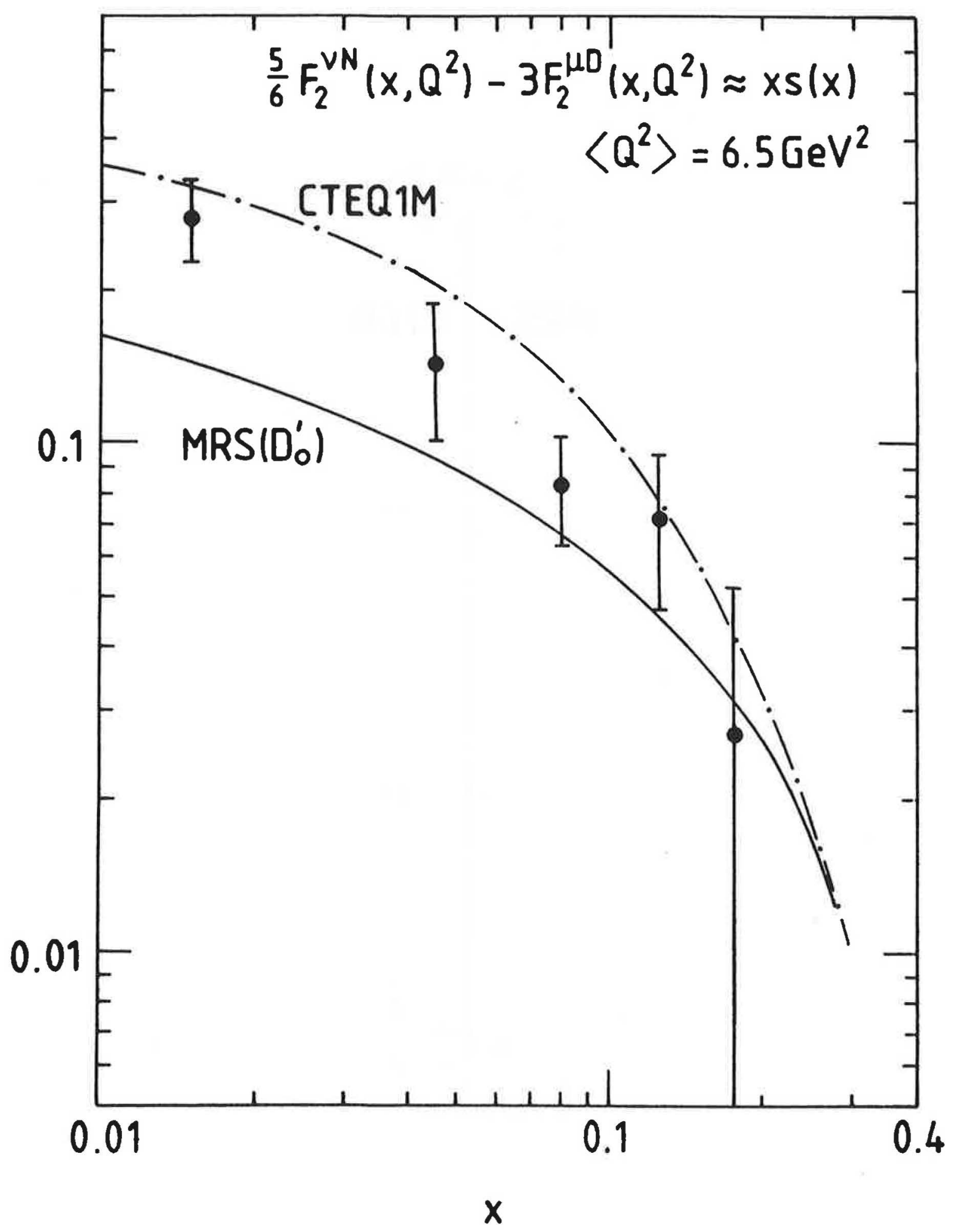

Fig. 8 


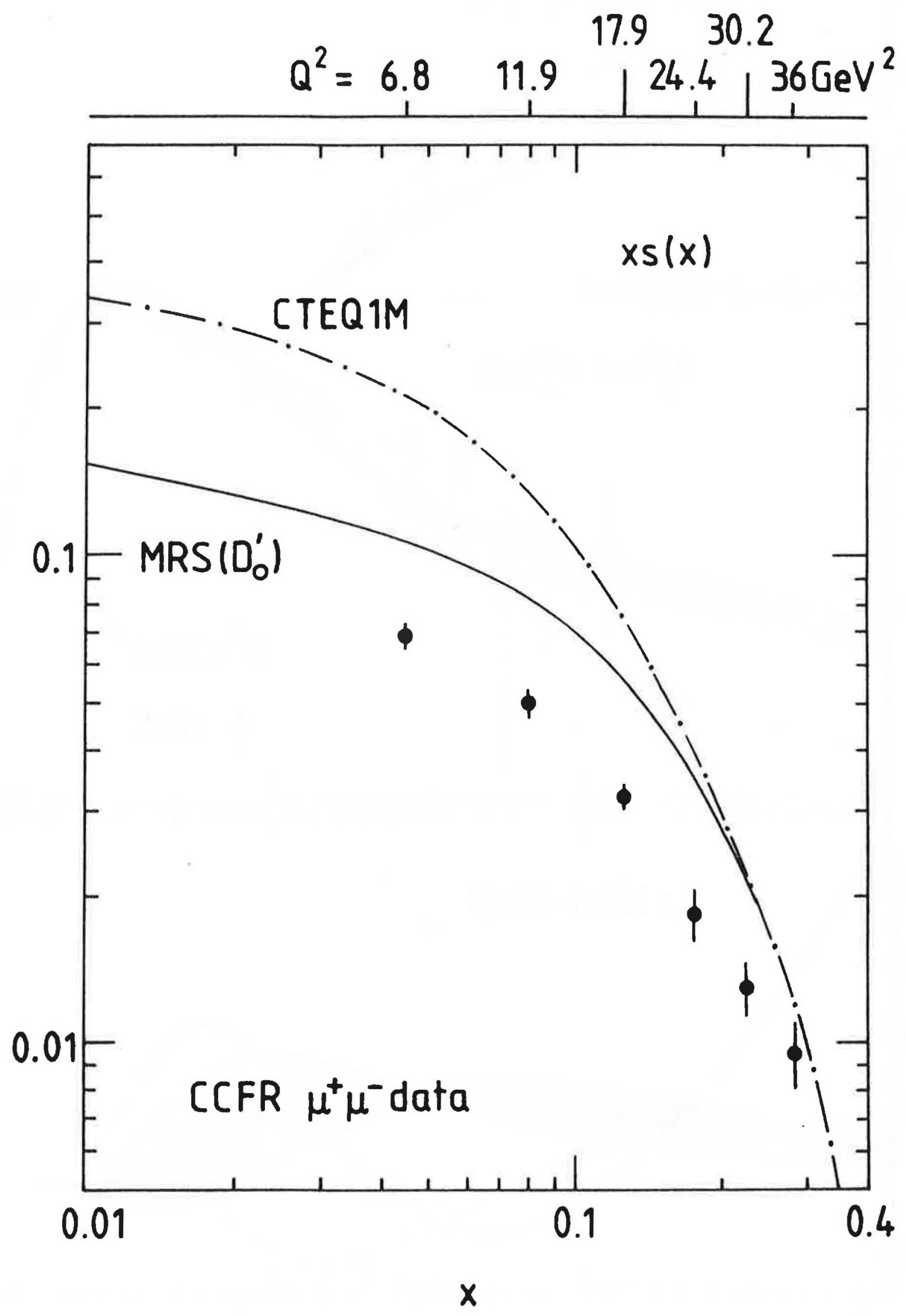

Fig. 9 


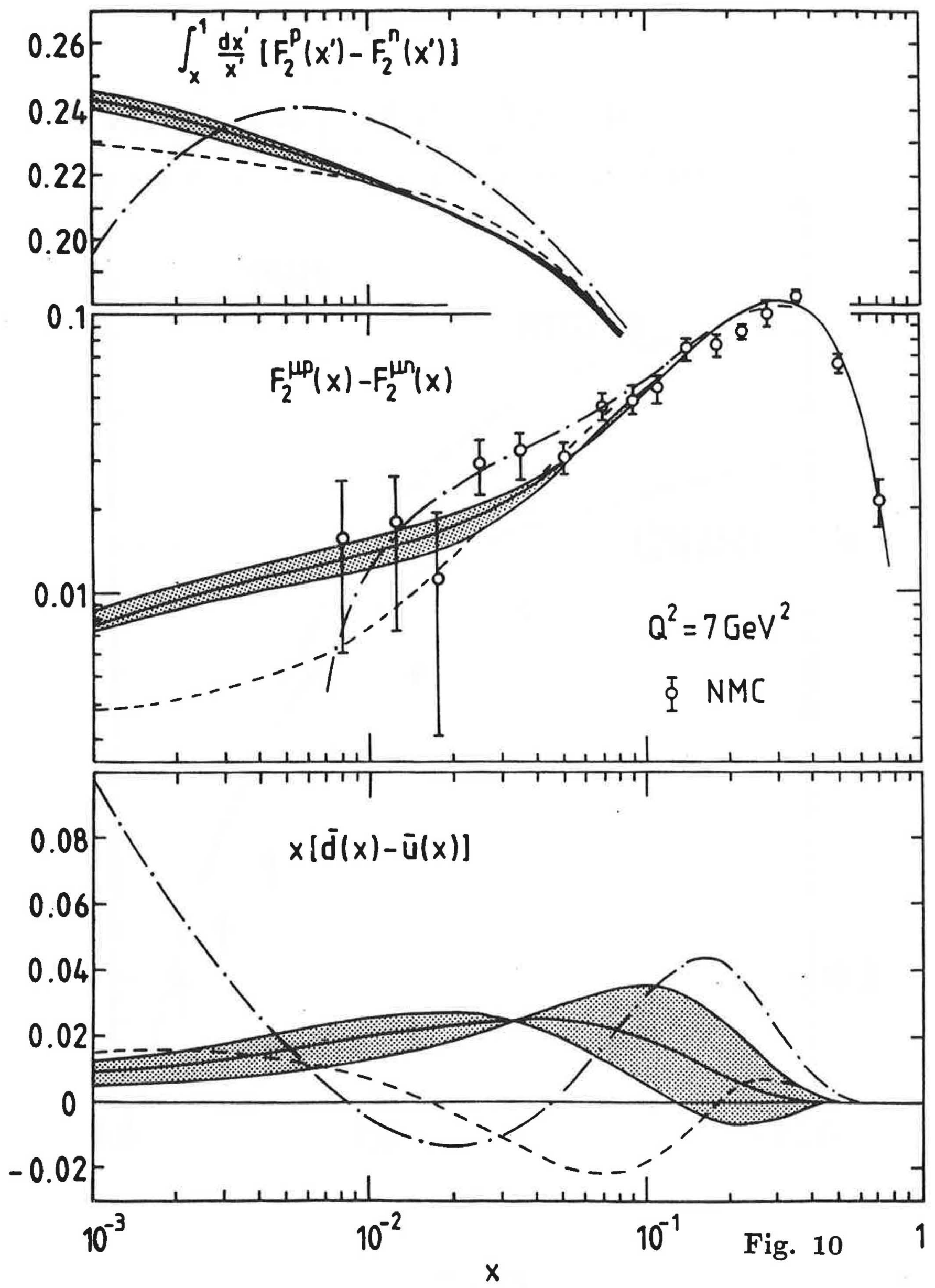




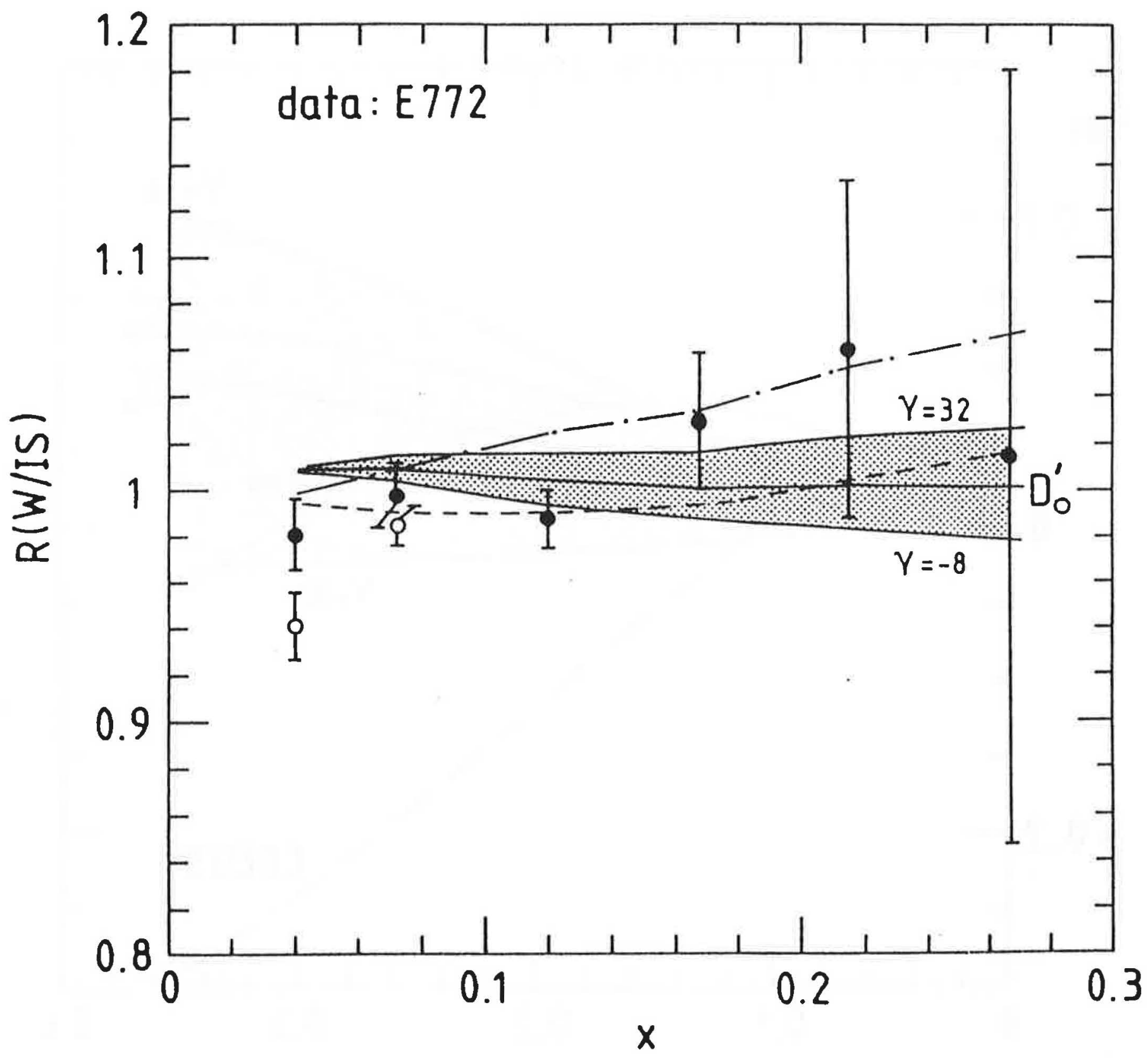

Fig. 11 


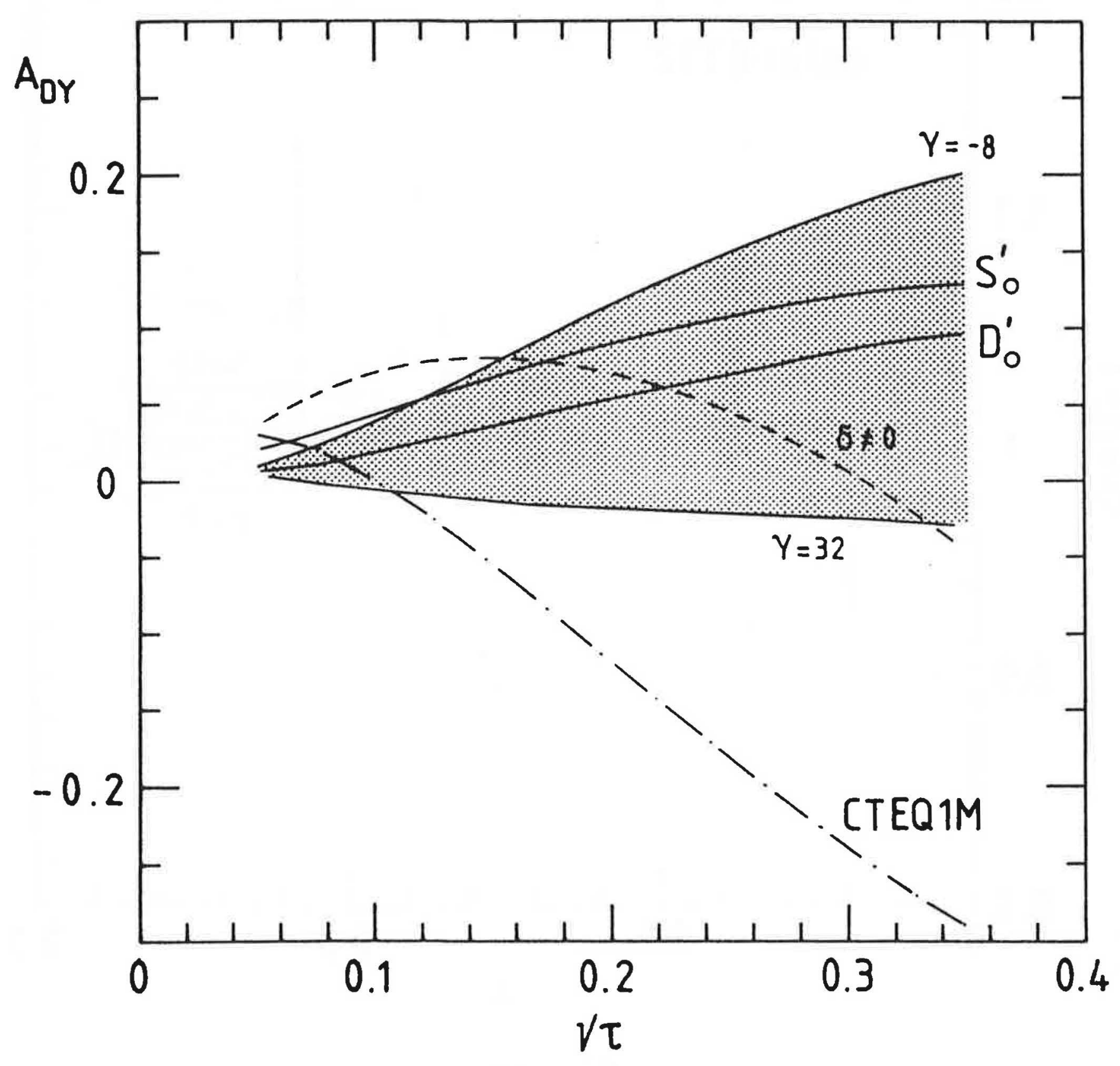

Fig. 12 


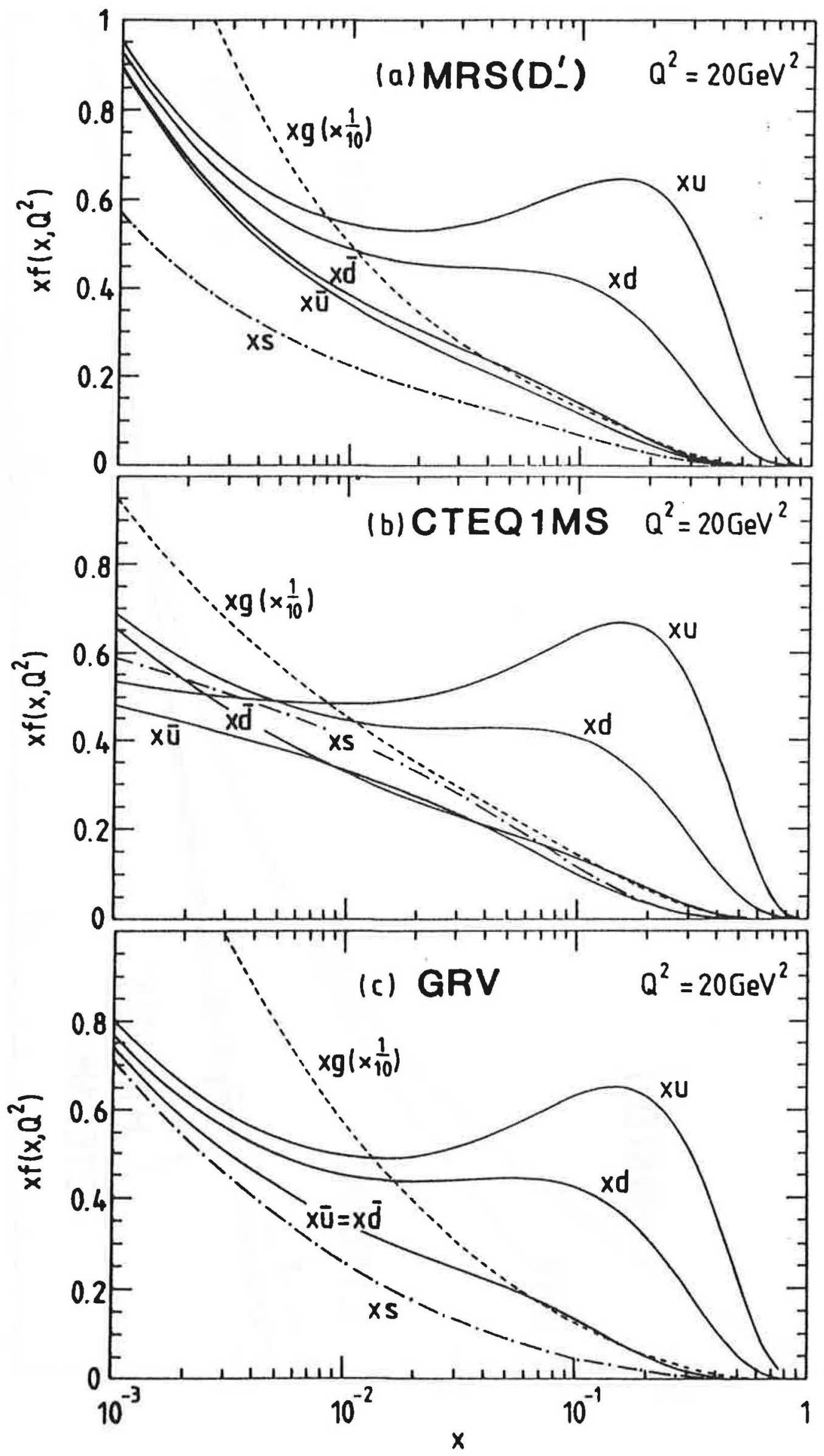

Fig. 13 


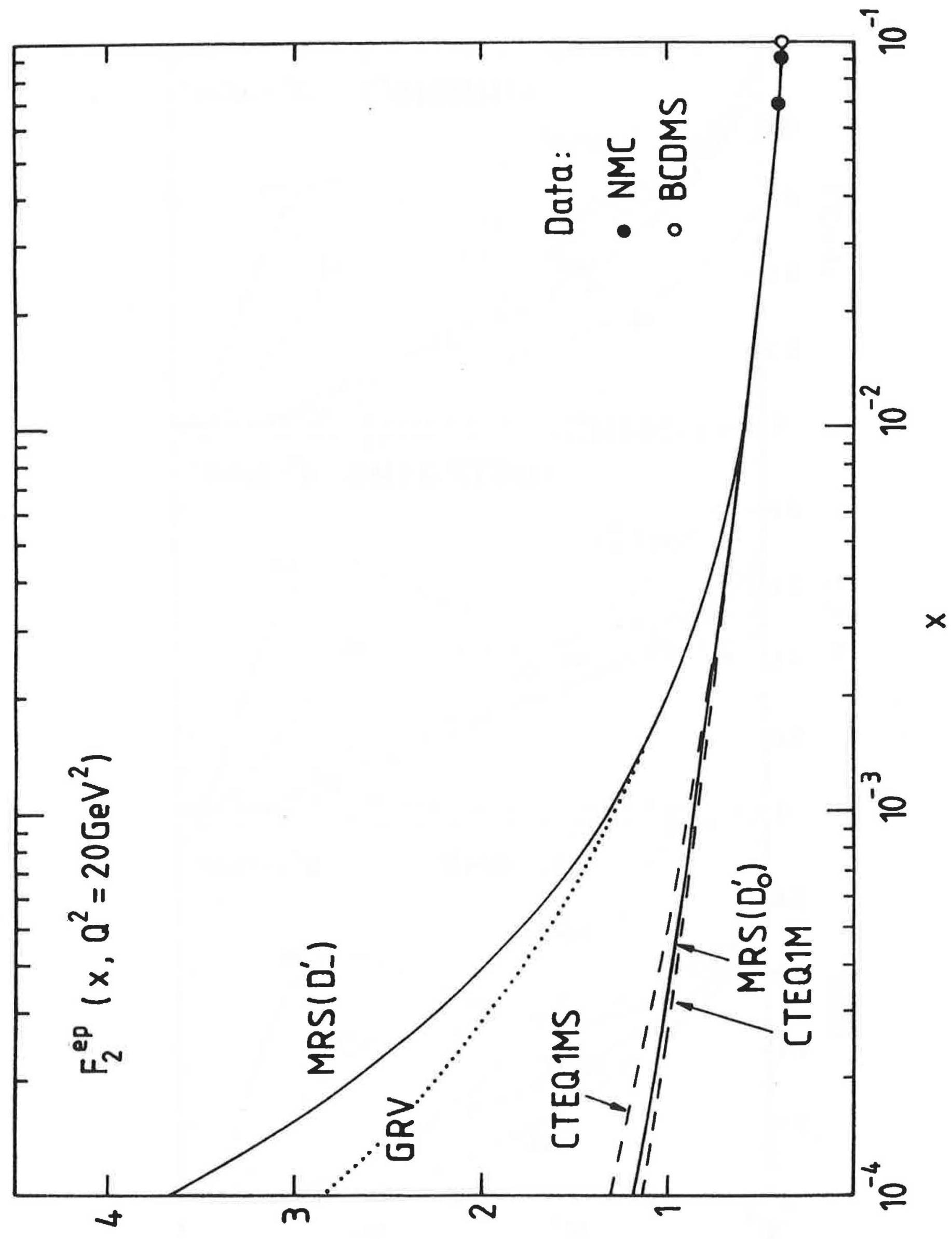

Fig. 14 

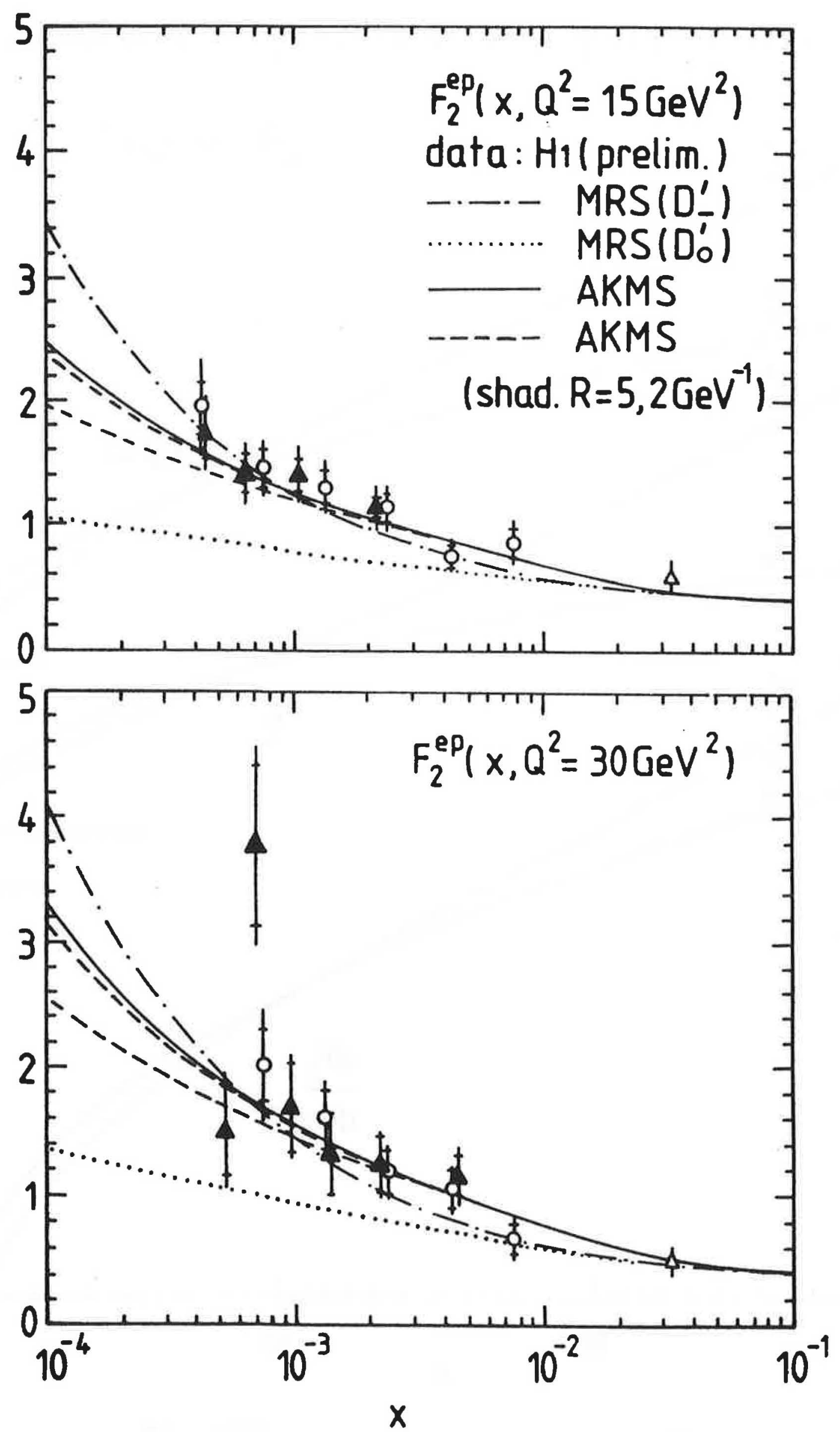

Fig. 15 


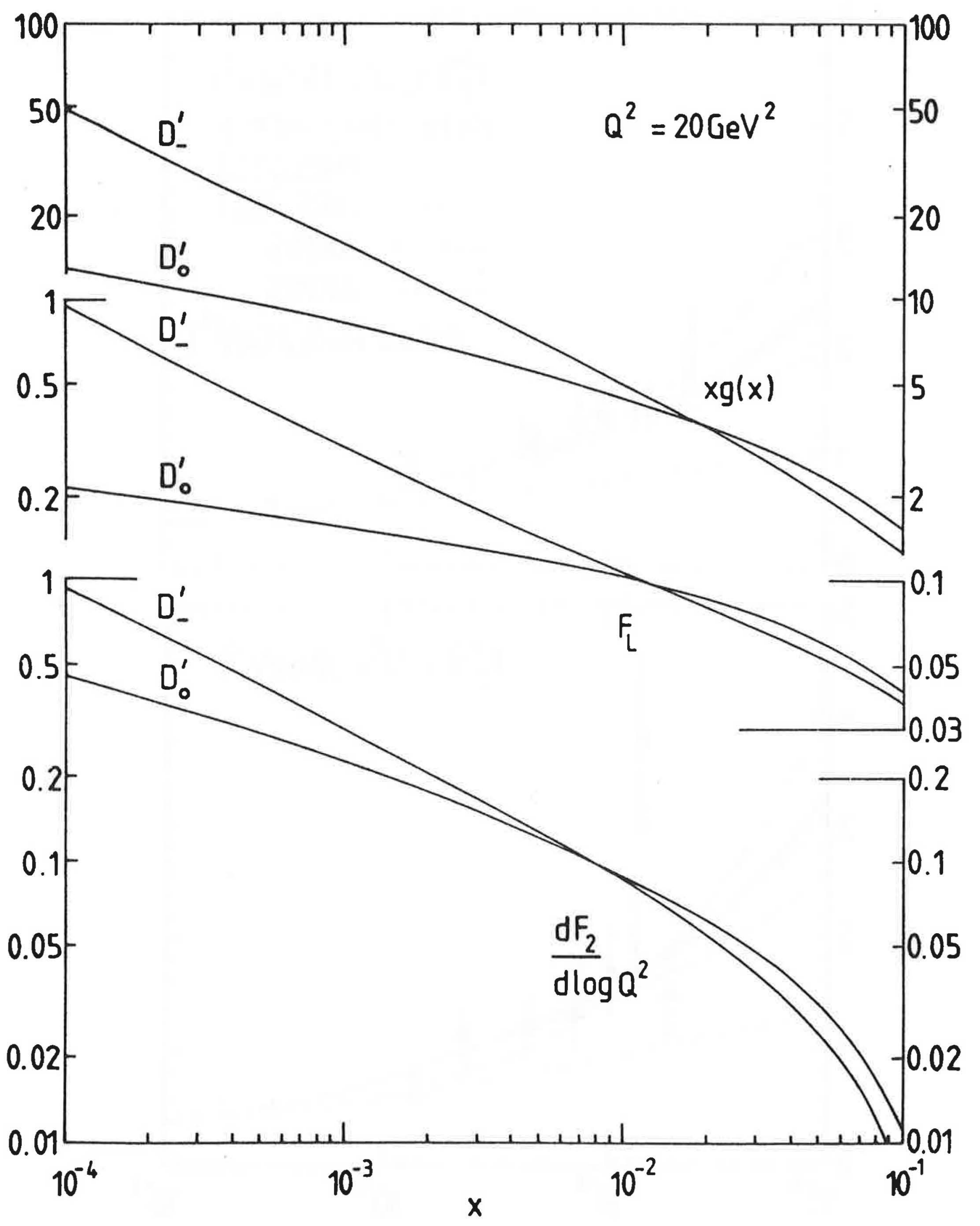

Fig. 16 
